\title{
Immunization expands B cells specific to HIV-1 V3 glycan in mice and macaques
}

Amelia Escolano ${ }^{1,11}$, Harry B. Gristick ${ }^{2,11}$, Morgan E. Abernathy², Julia Merkenschlager ${ }^{1}$, Rajeev Gautam³ ${ }^{3}$, Thiago Y. Oliveira ${ }^{1}$, Joy Pai ${ }^{1}$, Anthony P. West Jr ${ }^{2}$, Christopher O. Barnes ${ }^{2}$, Alexander A. Cohen ${ }^{2}$, Haoqing Wang ${ }^{2}$, Jovana Golijanin ${ }^{1}$, Daniel Yost ${ }^{1}$, Jennifer R. Keeffe ${ }^{2}$, Zijun Wang ${ }^{1}$, Peng Zhao ${ }^{4}$, Kai-Hui Yao' ${ }^{1}$, Jens Bauer ${ }^{1}$, Lilian Nogueira ${ }^{1}$, Han Gao², Alisa V. Voll², David C. Montefiori ${ }^{5}$, Michael S. Seaman ${ }^{6}$, Anna Gazumyan ${ }^{1}$, Murillo Silva ${ }^{7}$, Andrew T. McGuire ${ }^{8,9}$, Leonidas Stamatatos ${ }^{8,9}$, Darrell J. Irvine ${ }^{7}$, Lance Wells ${ }^{4}$, Malcolm A. Martin ${ }^{3}$, Pamela J. Bjorkman²* \& Michel C. Nussenzweig ${ }^{1,10 *}$

Broadly neutralizing monoclonal antibodies protect against infection with HIV-1 in animal models, suggesting that a vaccine that elicits these antibodies would be protective in humans. However, it has not yet been possible to induce adequate serological responses by vaccination. Here, to activate B cells that express precursors of broadly neutralizing antibodies within polyclonal repertoires, we developed an immunogen, $\mathrm{RCl}$, that facilitates the recognition of the variable loop 3 (V3)-glycan patch on the envelope protein of HIV-1. RCl conceals non-conserved immunodominant regions by the addition of glycans and/or multimerization on virus-like particles. Immunization of mice, rabbits and rhesus macaques with RC1 elicited serological responses that targeted the V3-glycan patch. Antibody cloning and cryo-electron microscopy structures of antibody-envelope complexes confirmed that immunization with RC1 expands clones of B cells that carry the anti-V3-glycan patch antibodies, which resemble precursors of human broadly neutralizing antibodies. Thus, RC1 may be a suitable priming immunogen for sequential vaccination strategies in the context of polyclonal repertoires.

Single-cell antibody cloning from human donors who are infected with HIV-1 revealed that broadly neutralizing antibodies (bNAbs) have undergone unusually extensive somatic mutations ${ }^{1-4}$. Moreover, the high degree of somatic mutations is essential for binding to the native HIV-1 envelope (Env) spike and for the neutralizing activity of bNAbs ${ }^{5}$. The accumulation of large numbers of mutations suggests that bNAbs evolve in response to iterative rounds of somatic hypermutation and selection in germinal centres ${ }^{6}$. Studies in humans revealed that this occurs in response to viral escape variants that arise from antibody pressure ${ }^{4}$. Together, these observations suggest that vaccination to elicit bNAbs requires a series of sequential immunogens starting with an immunogen that induces the expansion ${ }^{7}$ of B lymphocytes expressing appropriate germline precursors ${ }^{8}$.

Sequential immunization to guide the development of bNAbs was demonstrated in genetically modified mice that carry inferred germline precursors of human bNAbs ${ }^{8,9}$. However, the priming immunogens that were used to initiate the response failed to activate and expand $B$ cells that expressed inferred bNAb precursors in animals with polyclonal antibody repertoires. Thus, a goal of HIV-1 vaccine development has been to design immunogens that recruit B cells that express bNAb precursors into germinalcentre reactions in animals with polyclonal repertoires.

The germline-targeting approach to immunogen design focuses on producing immunogens that bind with high affinity to specific bNAb precursors-the rationale being that the recruitment of B cells to germinal centres is in part dependent on receptor affinity for antigen ${ }^{10-14}$. However, this methodology effectively limits the repertoire of recruited $B$ cells qualitatively and quantitatively. Moreover, it fails to account for the findings that each germinal centre accommodates different founder
B cells with a wide range of affinities, and that entry into the germinal centre is limited by competition and not absolute affinity ${ }^{7,10}$.

Here we describe RC1, an immunogen designed to recruit and expand diverse V3-glycan-specific B cells by improving accessibility of the V3-glycan patch epitope, which includes a group of highmannose and complex-type $N$-glycans that surround V3 (Asn residues in gp120: N133, N137, N156, N295, N301, N332, N339, N385 and $\mathrm{N} 392)^{15}$. bNAbs that target this site, including PGT12 $1^{16}, 10-1074^{17}$ and $\mathrm{BG} 18^{18}$, reach through these glycans using elongated heavy-chain complementarity-determining region (CDR) 3 (CDRH3) loops and portions of light-chain CDRs 1 and 3 (CDRL1 and CDRL3) to contact the highly conserved GDIR (G324-D325-I326-R327) motif at the base of $\mathrm{V}^{19}$. Here we show that $\mathrm{RC} 1$ activates and expands a diverse group of B cells expressing antibodies that resemble human V3-glycan patch bNAb precursors in mice, rabbits and rhesus macaques.

RC1 facilitates antibody binding to the V3-glycan patch $\mathrm{RC} 1$ was designed using $11 \mathrm{MUTB}^{20}$, a modified native-like Env trimer (SOSIP.664) derived from the clade A/E BG505 Env ${ }^{21}$, as a template. Compared to BG505, 11MUTB includes substitutions in V1 and lacks potential $N$-linked glycosylation sites (PNGSs) at N133 and N13720 (Fig. 1a). We reasoned that removal of the N156 PNGS (N156Q) to create $\mathrm{RC} 1$ would facilitate recognition of the V3-glycan patch by increasing accessibility of V1 residues that interact with V3-glycan bNAbs ${ }^{22,23}$. Consistent with this idea, the absence of the N156 PNGS enhances neutralization by PGT121 and 10-1074, whereas the absence of other glycans, such as N301 or N137, reduces neutralization (Extended Data Fig. 1a). In addition, we hypothesized that removal of the N156 glycan, which includes negatively charged terminal sialic acids ${ }^{22,24}$, would

\footnotetext{
${ }^{1}$ Laboratory of Molecular Immunology, The Rockefeller University, New York, NY, USA. ${ }^{2}$ Division of Biology and Biological Engineering, California Institute of Technology, Pasadena, CA, USA. ${ }^{3}$ Laboratory of Molecular Microbiology, National Institute of Allergy and Infectious Diseases, National Institutes of Health, Bethesda, MD, USA. ${ }^{4}$ Complex Carbohydrate Research Center, University of Georgia, Athens, GA, USA. ${ }^{5}$ Duke Human Vaccine Institute, Duke University Medical Center, Durham, NC, USA. ${ }^{6}$ Center for Virology and Vaccine Research, Beth Israel Deaconess Medical Center, Boston, MA, USA. ${ }^{7}$ David H. Koch Institute for Integrative Cancer Research, Massachusetts Institute of Technology (MIT), Cambridge, MA, USA. ${ }^{8}$ Vaccine and Infectious Disease Division, Fred Hutchinson Cancer Research Center, Seattle, WA, USA. ${ }^{9}$ Department of Global Health, University of Washington, Seattle, WA, USA. ${ }^{10}$ Howard Hughes Medical Institute, The Rockefeller University, New York, NY, USA. ${ }^{11}$ These authors contributed equally: Amelia Escolano, Harry B. Gristick. *e-mail: bjorkman@caltech.edu; nussen@rockefeller.edu
} 

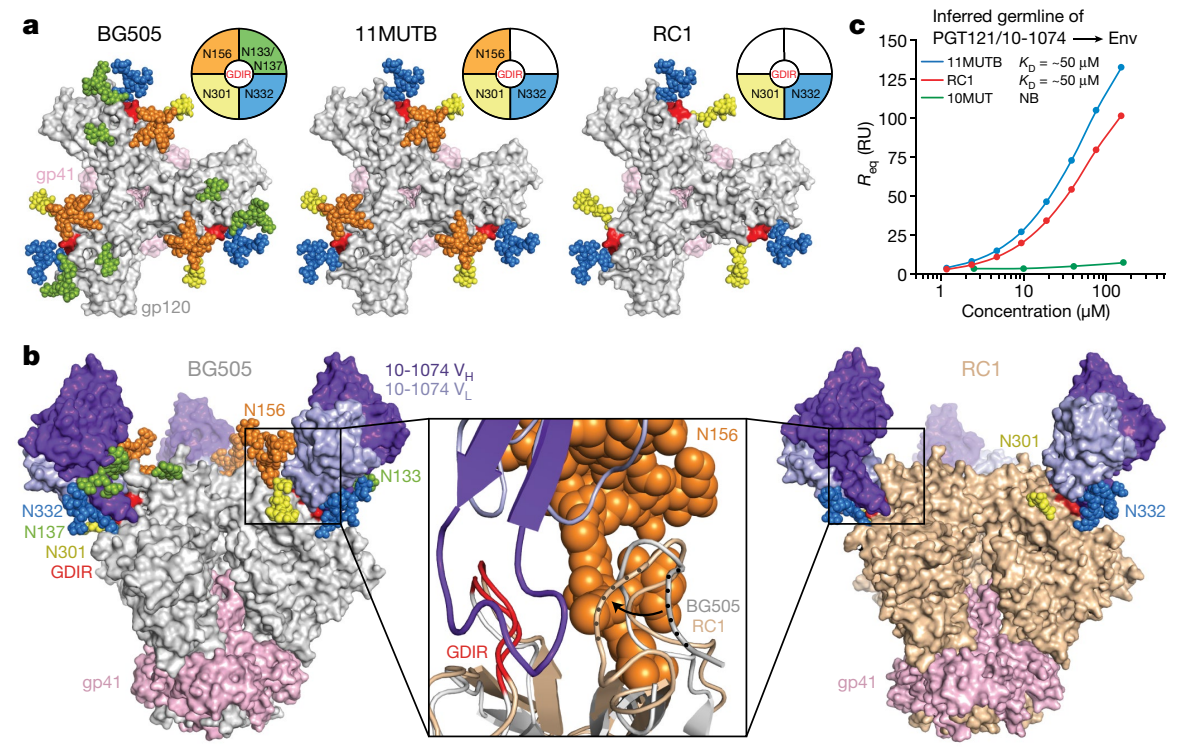

Fig. 1 Characterization of the RC1 immunogen. a, $N$-glycans (coloured spheres) and GDIR motifs (red surfaces) mapped onto BG505 (Protein Data Bank (PDB) code 5T3Z) (N137 glycan from PDB 5FYL) in the topdown orientation. $\mathbf{b}$, Left and right, side views of structures of BG505 and RC1 complexed with 10-1074 (glycan atoms are coloured spheres). Middle, superimposition of the boxed regions with protein in cartoon representations. Dark and light purple, $10-1074 \mathrm{~V}_{\mathrm{H}}$ and $\mathrm{V}_{\mathrm{L}}$, respectively; red, GDIR; wheat, other portions of RC1; grey, BG505; orange spheres, N156 glycan. Regions of V1 showing displacement (gp120 residues 139-140) are indicated by dots and an arrow. c, Surface plasmon resonance (SPR) data for PGT121/10-1074 binding to Env trimers. NB, no binding above background; RU, response units. Representative plot from three independent experiments.

the N156 glycan from 11MUTB, because removal of a nearby glycan at N301 that is also part of the glycan patch $(11$ MUTB $\Delta 301)$ (Extended Data Table 2) failed to induce detectable serological responses (Fig. 2h). We conclude that, in contrast to 11 MUTB and 11 MUTB $\triangle 301, \mathrm{RC} 1$ elicits V3-glycan-specific serological responses in wild-type mice.

To reduce antibody responses to off-target epitopes ${ }^{26-29}$ and further focus responses on the V3-glycan patch, we produced an RC1 variant, RC1-4fill, by introducing PNGSs to add glycans to gp120 positions 230, 241, 289 and 344 (Extended Data Fig. 4). Compared with RC1, RC1-4fill elicited serological responses that were more focused on the V3-glycan patch in wild-type mice (Fig. 2i). We conclude that RC1-4fill focuses antibody responses to the V3-glycan patch. 10-1074 CDRH3, allowing for increased interactions between RC1 and 10-1074 (Fig. 1b).

Despite structural changes in V1-which resulted from deletion of the N156 glycan (Fig. 1b) - the inferred germline of PGT121/ $10-1074^{17}$ bound to RC1 and 11MUTB with similar affinities (dissociation constant $\left(K_{\mathrm{D}}\right)$ values of approximately $50 \mu \mathrm{M}$; Fig. $1 \mathrm{c}$ ), and priming immunizations with RC1 and 11MUTB elicited comparable V3-glycan-specific serological responses in knock-in mice that carried genes encoding the inferred germline of PGT121/10-1074 ${ }^{9}$ (Fig. 2a-c). Thus, RC1 exhibited structural changes resulting from N156 glycan deletion that did not affect its affinity for the inferred germline of PGT121/10-1074.

\section{RC1 elicits V3-glycan antibodies in wild-type mice}

To determine whether RC1 can activate B cells carrying antibodies that are specific to the $\mathrm{V} 3$-glycan patch in wild-type mice, we immunized C57BL/6J mice once with RC1 or 11MUTB (Fig. 2a). 11MUTB did not produce a measurable serological response, but RC1-immunized mice showed reproducible anti-V3-glycan-patch-specific serological responses, as shown by enzyme-linked immunosorbent assays (ELISAs) in which we compared binding to $\mathrm{RC} 1$ and a mutant variant of RC1 (RC1-glycanKO) that lacks two V3 PNGSs (N301 and N332) that are critical for human V3-glycan patch bNAbs (Fig. 2d-g, Extended Data Table 2). Moreover, serum from the RC1-immunized mice crossreacted with $11 \mathrm{MUTB}$ but not with the more native-like Env $10 \mathrm{MUT}^{20}$ or with BG505 (Extended Data Fig. 3a). The improved immunogenicity of the V3-glycan patch epitope of RC1 results from specific removal of

\section{Expansion of V3-glycan-specific B cells in mice}

To further characterize the humoral responses elicited by RC1 and RC1-4fill, we sequenced antibody genes from single B cells from the germinal centre that bound to RC1 but not RC1-glycanKO (Extended Data Fig. 3b). All analysed RC1- and RC1-4fill-immunized mice showed expansion of B cell clones in the germinal centre (Fig. 2a, j). The expanded clones predominantly expressed heavy-chain $\mathrm{V}$ genes $\mathrm{V}_{\mathrm{H}} 5-6$, $\mathrm{V}_{\mathrm{H}}$ 9-3 and $\mathrm{V}_{\mathrm{H}} 2-9$, and $\kappa$ light-chain genes $\mathrm{V}_{\mathrm{K}}$ 3-4 and $\mathrm{V}_{\mathrm{K}}$ 14-111 (Fig. 2j, Extended Data Table 4 and Supplementary Tables 1, 2). The CDRH3 sequences in expanded clones showed similarities to human V3-glycan patch bNAbs, such as Tyr-rich or RXY motifs and longer-than-average CDRH3 sequences (Extended Data Table 4 and Supplementary Table 1). Consistent with a single immunization, the $V_{H}$ genes of the expanded clones had an average of 3.2 nucleotide mutations (Fig. $2 \mathrm{k}$ and Supplementary Table 1).

We mapped the target sites of these antibodies by ELISA against RC1 and $\mathrm{RC} 1$ mutant proteins. A diverse group of monoclonal antibodies showed V3-glycan-patch-specific binding (Fig. 2l). Further characterization of two mouse (MUR) antibodies, Ab275 MUR and Ab276 $6_{\text {MUR, }}$ showed that they bound to the V3-glycan patch of RC1 (Ab275 MUR, $K_{\mathrm{D}} \approx 30 \mathrm{nM}$ ) in a GDIR- and N301-glycan-dependent manner, and both retained binding to $11 \mathrm{MUTB}\left(\mathrm{Ab} 275_{\mathrm{MUR}}, K_{\mathrm{D}} \approx 230 \mathrm{nM}\right)-$ demonstrating accommodation of the N156 glycan-whereas neither antibody bound to BG505 or a peptide that covers the crown of the V3 loop (Fig. 2m, Extended Data Table 2, Extended Data Fig. 3c, d). 

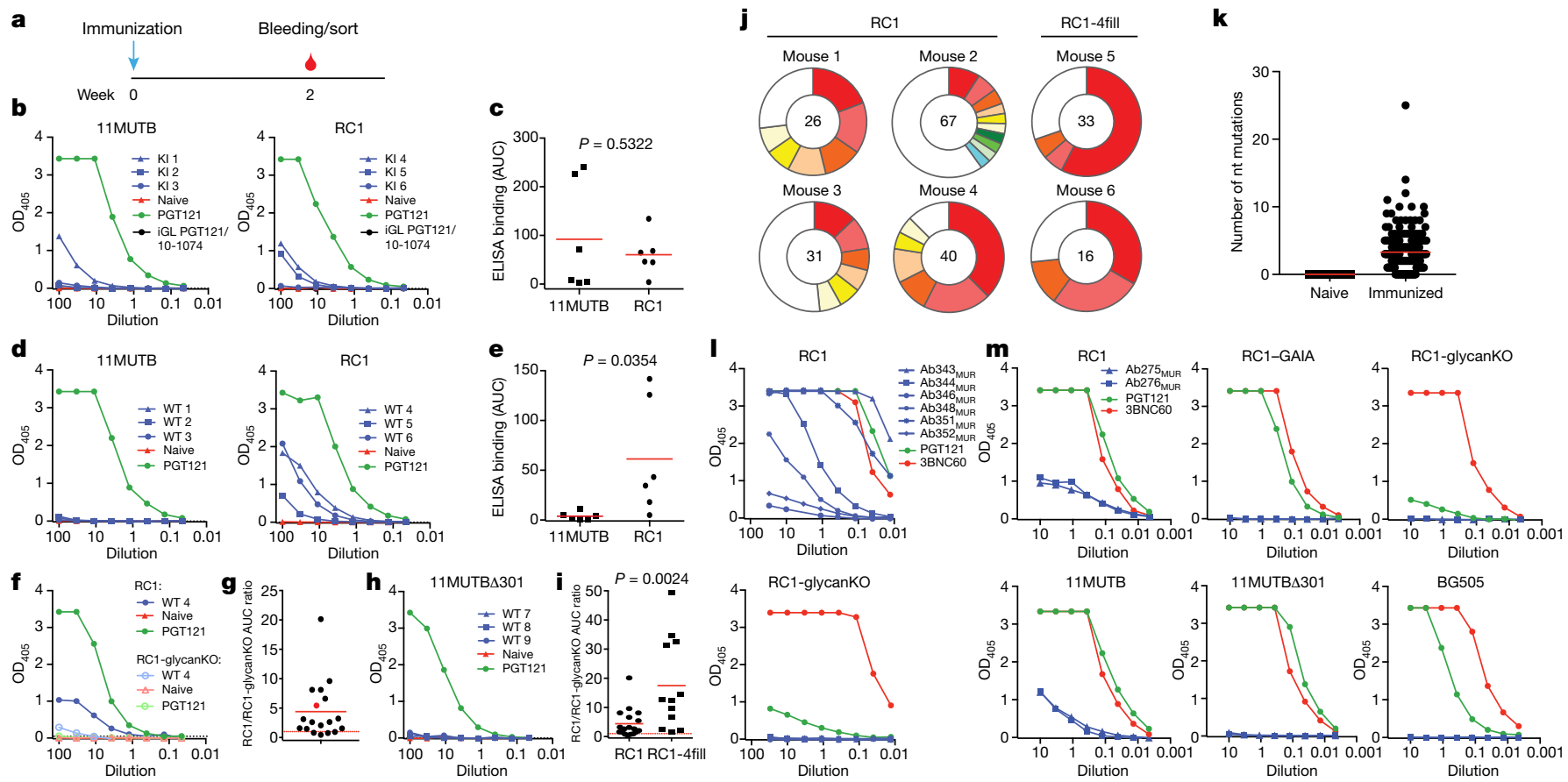

Fig. 2 | Wild-type mouse immunization with RC1 elicits V3-glycan patch antibodies. a, Immunization protocol. $\mathbf{b}, \mathbf{d}, \mathbf{f}, \mathbf{h}$, Representative ELISAs showing serum binding to indicated immunogens. Controls include naive serum (red), purified PGT121 (green) and inferred germline (iGL) of PGT121/10-1074 (black). OD 405 , optical density at $405 \mathrm{~nm}$. b, The inferred germline of PGT121 knock-in (KI) mice ${ }^{9}$. d, f, h, Wild-type (WT) mice. c, e, Area under the curve (AUC) for ELISAs in $\mathbf{b}$ and $\mathbf{d}$, respectively, but combined results from two experiments using three or four mice each. Each dot represents serum from one mouse. f, Binding to RC1 and RC1glycanKO. g, Ratio of the AUC for RC1 and RC1-glycanKO ELISAs from seven experiments with two or three mice immunized with RC1.

Red dot corresponds to mouse WT 4 in f. i, Ratio of the AUC for RC1 and

RC1-glycanKO ELISAs for wild-type mice immunized with RC1 (seven experiments) or RC1-4fill (five experiments). j, Pie charts show clonal expansion of RC1-binding B cells in the germinal centre. Coloured slices are proportional to the number of clonal relatives. White indicates single $\mathrm{IgV}_{\mathrm{H}}$ sequences. The number of heavy chains analysed is indicated in the centre. $\mathbf{k}$, IgH nucleotide (nt) mutations from naive and $\mathrm{RC} 1$ immunized mice in $\mathbf{j}$. l, ELISA binding of representative monoclonal antibodies from RC1-immunized mice to RC1 and RC1-glycanKO. m, ELISA binding of Ab275 MUR and Ab276 MUR to indicated Env proteins. c, e, i, Unpaired $t$-tests. $\mathbf{c}, \mathbf{e}, \mathbf{g}, \mathbf{i}, \mathbf{k}$, Data are mean and each dot is an individual mouse $(\mathbf{c}, \mathbf{e}, \mathbf{g}, \mathbf{i})$ or an individual sequence $(\mathbf{k})$.

Acquired mutations were essential for binding, because RC1 did not bind to the Ab276 ${ }_{\mathrm{MUR}}$-reverted inferred germline (Extended Data Fig. 3e). Consistent with a single immunization, neither Ab275 MUR nor $\mathrm{Ab} 276_{\mathrm{MUR}}$ showed detectable neutralizing activity against a panel of tier $1 \mathrm{~B}$ and tier $2 \mathrm{HIV}-1$ isolates in assays using the TZM-bl reporter cell line. We conclude that RC1 and RC1-4fill expand mouse B cell clones expressing antibodies that target the V3-glycan patch.

\section{V3-glycan antibody responses in rabbits and macaques}

To enhance potential avidity effects and limit exposure of off-target epitopes at the Env base, we multimerized RC1-4fill on virus-like particles (VLPs) using the SpyTag-SpyCatcher system ${ }^{30,31}$ (Fig. 3a, b). VLPs were used to prime rabbits and rhesus macaques. Single immunizations of 4 rabbits and 16 macaques with VLP-RC1-4fill elicited serological responses that were partially specific for the V3-glycan patch in all animals (Fig. 3c-f, Extended Data Fig. 5a). Serum from macaques primed with VLP-RC1-4fill showed sequentially reduced binding to the more native-like immunogens $11 \mathrm{MUTB}$ and $10 \mathrm{MUT}^{20}$ (Extended Data Fig. 5b) and no neutralizing activity against a small panel of HIV-1 isolates that included fully glycosylated tier 2 and glycan-deleted viruses (Extended Data Table 3). Thus, VLP-RC1-4fill elicited robust serological responses that mapped in part to the V3-glycan patch in rabbits and rhesus macaques.

To further characterize responses elicited by VLP-RC1-4fill in macaques, we purified germinal centre $B$ cells from the draining lymph nodes that bound to RC1 but not to RC1-glycanKO by flow cytometry $\left(\mathrm{RC1}^{+} \mathrm{RC1} 1\right.$-glycanKO $\left.{ }^{-}\right)$. Whereas $\mathrm{RC}^{+}$cells were absent from germinal centres of naive macaques, $\mathrm{RC}^{+} \mathrm{RC} 1$-glycan $\mathrm{KO}^{-}$germinal centre $\mathrm{B}$ cells were found at an average frequency of $0.4 \%$ of germinal centre $\mathrm{B}$ cells in the lymph nodes in the four macaques analysed (Fig. 3g, h).

Antibody cloning from four immunized macaques revealed expanded $\mathrm{B}$ cell clones that used a variety of $\mathrm{V}_{\mathrm{H}}$ genes, as found for human V3-glycan patch bNAbs ${ }^{32}$, with an average of 5.6 nucleotide somatic mutations (Fig. 3i, j and Supplementary Table 3). Most characterized human V3-glycan patch bNAbs contain a $\lambda$ light chain ${ }^{18,33}$. Analysis of $\lambda$ genes revealed that macaque RC1-binding cells preferentially used gene segments $\mathrm{V}_{\mathrm{L}} 132$ (91\% nucleotide sequence identity to $\mathrm{V}_{\mathrm{L}}$ 2-8 germline gene segments in PGT125-PGT128 and PGT130PGT131) and $V_{L} 124$ (94\% nucleotide sequence identity to the $V_{L} 3-21$ germline in PGT121-PGT123/10-1074) (Fig. 3k). In addition, 86\% of the $\lambda$ light chains had CDRL3 sequences that included an Asp-SerSer (DSS) motif present in the inferred germlines of PGT121-123 and 10-1074/PGT124 ${ }^{17}$ (Fig. 31 and Supplementary Table 4). This motif mutates to Asp-Ser-Arg (DSR) in the mature bNAbs, which is critical for the neutralization activity of PGT $121^{34}$. Thus, there is congruence between the sequence of precursors of human V3-glycan patch bNAbs and the antibodies expressed by macaque $\mathrm{B}$ cell clones that are elicited by priming with VLP-RC1-4fill.

We expressed 38 macaque germinal centre antibodies with CDRL3 sequences that resembled the CDRL3 sequences of inferred germline V3-glycan patch bNAbs (Supplementary Table 5). The CDRL3 sequences of 33 out of 38 antibodies contained a DSS motif and a Gln at position 89 (QXXDSS motif), which was also found in the CDRL3 sequence of the inferred germlines of PGT121-PGT123, 10-1074, PGT124 and BG18 $8^{17,18}$ (Extended Data Table 5). In addition, 5 out of 38 antibodies contained a Ser-Tyr-Ala-Gly (SYAG) motif, which is present in the CDRL3 sequences of the inferred germlines of PGT125PGT128, PGT130 and PGT131 (Extended Data Table 5). In total, 30 out of 33 QXXDSS motif-containing antibodies and 2 out of 5 SYAG motif-containing antibodies bound to the V3-glycan patch epitope, as 
a

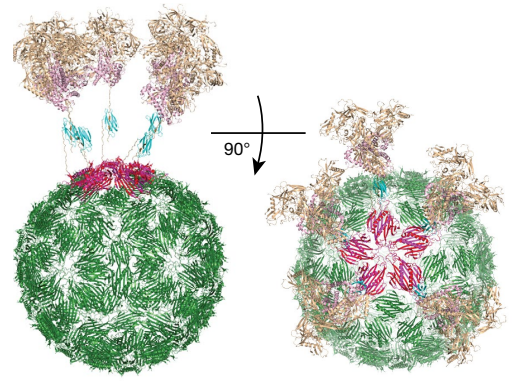

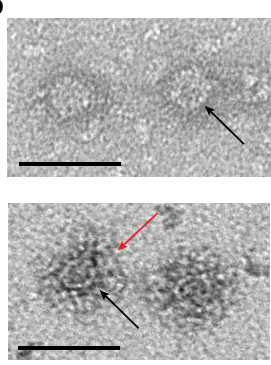

c

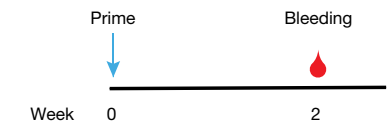

e

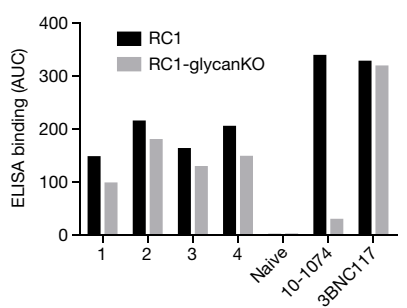

$\mathbf{g}$
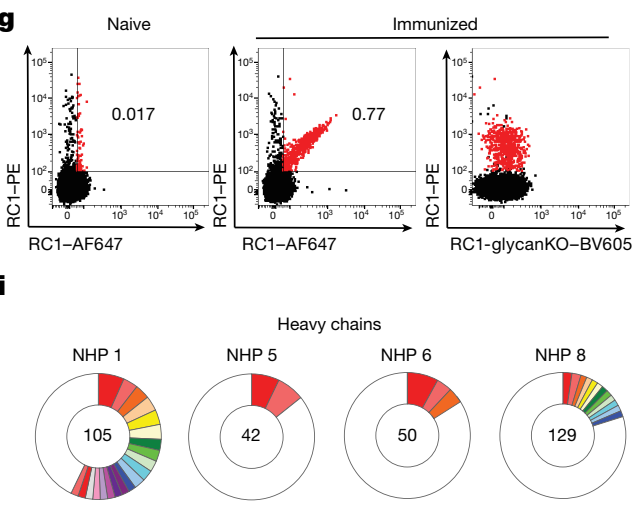

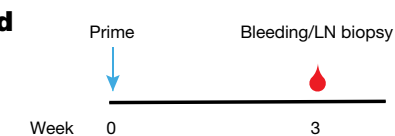

$\mathbf{f}$

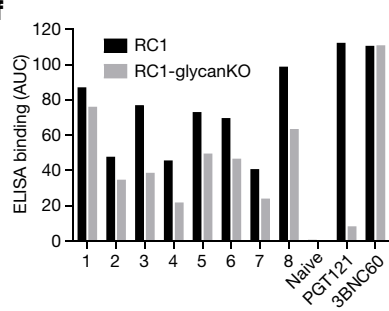

h

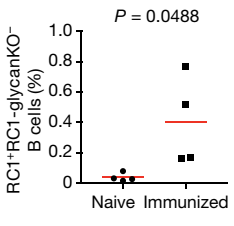

j

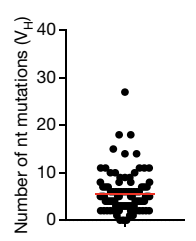

$\mathbf{k}$

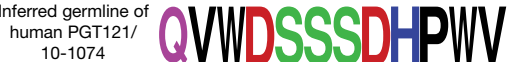

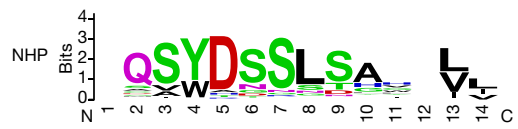

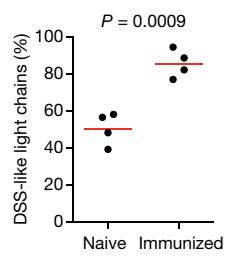

Fig. 3 Macaque immunization with RC1-4fill VLPs elicits anti-V3glycan patch antibodies that resemble inferred germlines of bNAbs. a, Model of VLP-RC1-4fill: RC1-4fill (wheat and pink), SpyTag (gold), SpyCatcher (cyan) and bacteriophage AP205 (green). b, Negativestain electron microscopy images comparing VLPs (top) and VLP-RC1 (bottom). Arrows indicate the VLP surface (black) and RC1 (red). Scale bars, $50 \mathrm{~nm}$. Representative image from three independent experiments. c, d, Immunization protocols for rabbits (c) and non-human primates (d). LN, lymph node. e, f, AUC for ELISAs with serum from four rabbits (e) and eight non-human primates (f) primed with VLP-RC1-4fill against RC1 (black) and RC1-glycanKO (grey). g, h, Flow cytometry plots showing frequency of B cells in the germinal centre that bind to RC1 but not to RC1-glycanKO. g, Representative flow cytometry plots. h, Quantification. $n=4$ naive and $n=4$ immunized non-human primates. i, Pie charts showing clonal expansion of RC1-binding $B$ cells in the germinal centre (see legend in Fig. 2j). j, $\operatorname{IgV}_{\mathrm{H}}$ mutations for the sequences of clones shown in $\mathbf{i}$ (Supplementary Table 3). $\mathbf{k}$, Logo plots comparing CDRL3 of inferred germline of PGT121/10-1074 and all IgL from B cells in the germinal centre shown in $\mathbf{i}$. $\mathbf{l}$, Fraction of CDRL3 sequences from $\mathbf{i}$ that show a DSS-like motif. h, $\mathbf{l}$, Unpaired $t$-test. $\mathbf{h}, \mathbf{j}, \mathbf{l}$, Data are mean and individual values.

determined by ELISA using RC1 and RC1-glycanKO with additional mutations in the GDIR sequence (RC1-glycanKO-GAIA) (Fig. 4a and Supplementary Table 5). In addition, the CDRL1 of all 38 macaque antibodies contained an Asn-Ile-Gly (NIG)-like motif present in the PGT121/10-1074 antibody (33 with a NIG motif, 4 with a DIG (AspIle-Gly) motif and 1 with a NLG (Asn-Leu-Gly) motif; Supplementary Table 5). The CDRH3 lengths of the 38 antibodies were relatively long (11-21 residues; average $=15.5$ residues) (Fig. 4b). Longer CDRH3 sequences were enriched in Tyr and/or Phe residues, similar to the long CDRH3 sequences found in human V3-glycan patch bNAbs ${ }^{16-18}$ (Supplementary Table 5). The antibody $\mathrm{V}_{\mathrm{H}}$ and $\mathrm{V}_{\mathrm{L}}$ genes included an average of 4.9 and 3.3 nucleotide mutations, respectively (Fig. 4c). Consistent with recruitment of antibodies with a range of affinities to germinal centres and their subsequent affinity maturation ${ }^{7,10}$, the inferred germline versions of the macaque antibodies showed lower affinities for RC1 than their mutated counterparts, ranging from levels that were below quantification to $K_{\mathrm{D}}$ values in the micromolar range (Extended Data Fig. 5c). Similarity between the macaque antibodies and PGT121/10-1074 was corroborated by ELISAs using an antiidiotypic antibody specific for the PGT121/10-1074 inferred germline antibody (Extended Data Fig. 5d). The inferred germline-reverted versions of 5 out of 11 macaque antibodies were recognized by the antiPGT121/10-1074 antibody (Extended Data Fig. 5d).

To further characterize the target site of the macaque antibodies, we performed ELISAs against additional proteins: RC1-glycanKO, RC1GAIA, RC1-glycanKO-GAIA, 11 MUTB $\Delta 301$, RC1 $\Delta 301$, RC1 $\Delta 332$, $11 \mathrm{MUTB}^{20}$ and BG505 (Fig. 4d, e, Extended Data Table 2). The ELISAs suggested four distinct RC1-binding patterns among antibodies that contained a CDRL3 QXXDSS motif (Fig. 4d) and another pattern among antibodies that contained a SYAG motif (Fig. 4e). Whereas all antibodies were glycan dependent as determined by no binding to RC1-glycanKO, they differed in binding to 11MUTB or 10MUT and dependence on GDIR and N301, N332 and N156 glycans (Fig. 4a, d, e). Although none of the antibodies recognized BG505, Ab933 ${ }_{\mathrm{NHP}}$ (where NHP denotes antibodies elicited in non-human primates), Ab936 $6_{\mathrm{NHP}}$ and $\mathrm{Ab} 1170_{\mathrm{NHP}}$ bound to $11 \mathrm{MUTB}$, indicating that they can accommodate the N156 glycan (Fig. 4d, e). Consistent with no binding to BG505, none of the antibodies exhibited neutralizing activity, and removal of the N133, N137 and N156 glycans did not render the BG505/T332N and JRCSF.JB viruses sensitive to neutralization (Extended Data Table 3b, c), suggesting that the lack of neutralization is not due to clashes with those glycans. We conclude that macaque immunization with VLP-RC1-4fill elicits V3-glycan-patch-specific antibodies that resemble precursors of human bNAbs that target this site.

\section{Cryo-EM structures of antibodies bound to RC1}

We determined structures of one mouse and two macaque Fabs complexed with RC1 using single-particle cryo-EM. Ab275 ${ }_{\text {MUR }}(4.4-\AA$ resolution) and $\mathrm{Ab} 874_{\mathrm{NHP}}(3.9 \AA$ ) (derived from the same clone as Ab876 $6_{\mathrm{NHP}}$ ) bound $\mathrm{RC} 1$ similarly to each other, consistent with their $69 \% \mathrm{~V}_{\mathrm{H}}$ domain amino acid sequence identity, whereas Ab897 $\mathrm{NHP}$ ( $4.4 \AA$ ) (related by $48 \%$ and $54 \% \mathrm{~V}_{\mathrm{H}}$ sequence identity to $\mathrm{Ab} 275_{\mathrm{MUR}}$ and Ab874 $4_{\mathrm{NH}}$, respectively) adopted a distinct angle of approach (Fig. 5a, Extended Data Fig. 5e).

All three Fabs in the RC1 complexes bound to the V3-glycan patch epitope and contacted the GDIR motif, but with different orientations and footprints from each other and from V3-glycan patch bNAbs (Fig. 5a, Extended Data Fig. 5e). 10-1074 contacts the conserved GDIR motif using CDRH3, CDRL1 and CDRL3 ${ }^{22}$ (Figs. 1c, 5a); Ab874 ${ }_{\mathrm{NHP}}$ and $\mathrm{Ab} 275_{\mathrm{MUR}}$ made GDIR contacts using $\mathrm{CDRH} 2$; whereas $\mathrm{Ab} 897_{\mathrm{NHP}}$ used CDRL1 and CDRL3 (Fig. 5a, b). In addition, Ab874 $4_{\text {HP }}$ and Ab897 ${ }_{\text {NHP }}$ contain the conserved CDRL3 QXXDSS motif, which makes contact with conserved regions of the $\mathrm{V} 3$-glycan epitope in mature bNAbs $^{22,35}$. Similar to mature V3-glycan patch bNAbs, Ab897 ${ }_{\text {HPP }}$ contains a substitution (Ser93Asn) within the QXXDSS motif that enables contacts with gp $120_{\mathrm{GDIR}}$, and also uses its conserved CDRL1 NIG motif to contact the V1 loop (Extended Data Fig. 5f). 

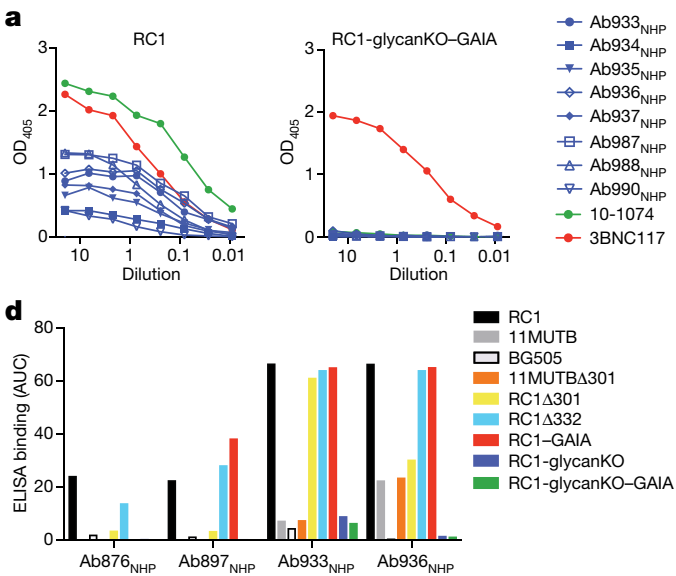
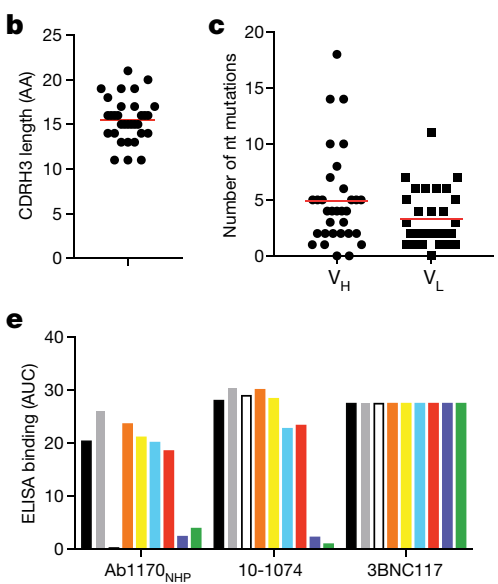

Fig. 4 Monoclonal antibodies from macaques bind to the V3-glycan patch. a, ELISA binding of representative macaque monoclonal antibodies to RC1 and RC1-glycanKO-GAIA. b, CDRH3 length of 32 V3-glycan-patchspecific monoclonal antibodies. AA, amino acids. c, Nucleotide mutations in $\operatorname{IgV}_{\mathrm{H}}$ and $\operatorname{IgV}_{\mathrm{L}}$ of 32 V3-glycan-patch-specific monoclonal antibodies. d, e, AUCs for ELISA binding of monoclonal antibodies to indicated proteins. Each AUC value corresponds to one ELISA curve. b, c, Data are mean and each dot is an individual sequence.
Ab275 ${ }_{\text {MUR }}$ and Ab874 $4_{\text {NHP }}$ also interacted with the N332 glycan, consistent with mature V3-glycan bNAbs (Fig. 5a, Extended Data Fig. 5e). However, in contrast to 10-1074-which interacts with the N332 glycan via its CDRL1, light-chain framework region 3 (FRWL3), $\mathrm{CDRH} 2$ and $\mathrm{CDRH}^{22}-\mathrm{Ab} 275_{\mathrm{MUR}}$ made contacts using CDRH2, whereas Ab874 $4_{\mathrm{NHP}}$ engaged the $\mathrm{N} 332$ glycan with $\mathrm{CDRH} 2$ and heavychain framework region 3 (FRWH3). We did not observe N332 glycan interactions in the Ab897 ${ }_{\mathrm{NHP}}-\mathrm{RC} 1$ structure. Despite reduced binding of $\mathrm{Ab} 275_{\mathrm{MUR}}, \mathrm{Ab} 876_{\mathrm{NHP}}$ (same clone as Ab874 $4_{\mathrm{NHP}}$ ) and $\mathrm{Ab} 897_{\mathrm{NHP}}$ to RC1 $\Delta 301$ (Fig. 2l), none of these Fabs showed interactions with the N301 glycan in our structures, suggesting that glycan heterogeneity obscures this interaction, and/or conformational heterogeneity in a V3-glycan patch that lacks this glycan diminishes binding ${ }^{36}$. We conclude that RC1 elicits V3-glycan-patch-targeting antibodies with distinct binding modes in animals with polyclonal antibody repertoires.

\section{Conclusions}

HIV-1 bNAbs develop in infected humans by sequential rounds of somatic mutation in response to a rapidly evolving pathogen ${ }^{4}$. Vaccination with a series of related antigens can reproduce this progression of events in genetically engineered mice that carry supraphysiological numbers of B lymphocytes that express the inferred germline precursors of bNAbs ${ }^{9}$. An important goal of HIV-1 vaccine design is to develop immunogens that initiate this response in organisms with polyclonal immune systems and then reproduce these responses in humans.

HIV-1 immunogen design has focused on increasing the affinity of candidate immunogens for specific inferred germline precursors of bNAbs, with the objective of recruiting a specific group of rare precursors into the germinal centre ${ }^{1}$. This approach typically fails to account for increases in apparent affinity produced by interactions between multimerized antigen and clusters of bivalent antigen receptors on the surface of a B cell. Moreover, entry into the germinal centre is primarily limited by competition $7,10,11,14$. Thus, the importance of affinity is relative, as shown by the observation that $\mathrm{B}$ cells bearing low-affinity receptors are frequently found in germinal centres under physiological conditions ${ }^{10,37}$, and by our finding that inferred germline precursors of macaque antibodies elicited by $\mathrm{RC} 1$ showed relatively low affinity for the immunogen.

The principles we used to produce RC1 did not take affinity for a germline B cell receptor into account. Instead, RC1 was designed to increase the number of $\mathrm{bNAb}$ progenitors that compete for entry into germinal centres by making the antigenic target site more available and facilitating binding to electrostatically neutral inferred germline precursors $^{25}$. In addition, VLP-RC1-4fill incorporates the idea that masking competing off-target epitopes ${ }^{26,29}$ by addition of glycans ${ }^{27}$ and tethering the bottom of the trimer to a VLP minimizes competition for entry into the germinal centre. a

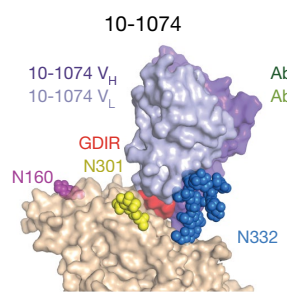

$\frac{1}{90 \%}$

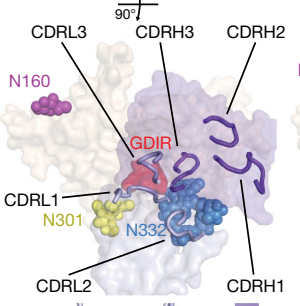

b

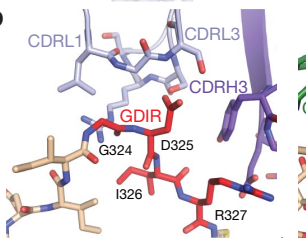

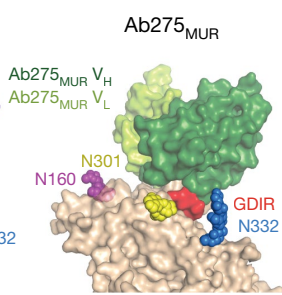
$\frac{1}{90^{\circ}}$

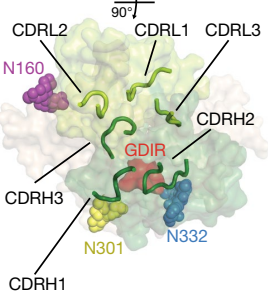

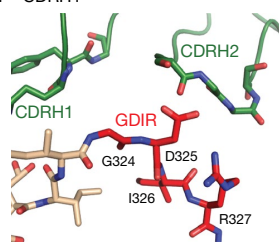
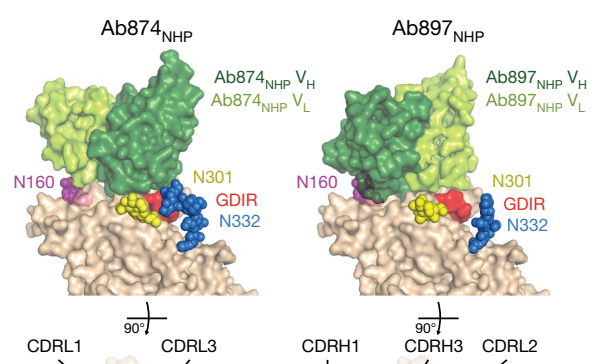

Fig. 5 | Structures of 10-1074 and elicited antibodies bound to RC1. a, Top, $\mathrm{V}_{\mathrm{H}}-\mathrm{V}_{\mathrm{L}}$ domains of 10-1074 and elicited antibodies bound to one protomer of RC1 (GDIR residues are red; glycans are coloured spheres). Bottom, antibody combining sites (CDRs shown as loops) mapped onto gp120 (glycans as coloured spheres; GDIR in red). $\mathbf{b}$, Comparisons of interactions of the GDIR motif with 10-1074 and elicited antibodies (colours as in $\mathbf{a}$ ). 
$\mathrm{RC} 1$ differs from other HIV-1 vaccine candidates in that it induces $B$ cells that express antibodies against a targeted epitope to undergo clonal expansion in germinal centres in animals with a fully polyclonal $B$ cell repertoire. In macaques, these B cells express antibodies that show sequence and structural similarities to inferred germline precursors of bNAbs that target the V3-glycan patch. Like the precursors of human bNAbs, they do not bind to wild-type Env or neutralize HIV$1^{5}$. Notably, biochemical and structural results showed that antibodies with distinct mechanisms of targeting the V3-glycan patch were elicited by $\mathrm{RC} 1$, increasing the probability that one or more might develop breadth and potency after boosting ${ }^{9}$. Thus, VLP-RC1-4fill is a suitable candidate immunogen for further evaluation in sequential vaccination strategies to elicit bNAbs.

\section{Online content}

Any methods, additional references, Nature Research reporting summaries, source data, statements of data availability and associated accession codes are available at https://doi.org/10.1038/s41586-019-1250-z.

Received: 10 December 2018; Accepted: 1 May 2019; Published online 29 May 2019.

1. McCoy, L. E. \& Burton, D. R. Identification and specificity of broadly neutralizing antibodies against HIV. Immunol. Rev. 275, 11-20 (2017).

2. West, A. P. Jr et al. Structural insights on the role of antibodies in HIV-1 vaccine and therapy. Cell 156, 633-648 (2014)

3. Kwong, P. D. \& Mascola, J. R. HIV-1 vaccines based on antibody identification, B cell ontogeny, and epitope structure. Immunity 48, 855-871 (2018).

4. Bonsignori, M. et al. Antibody-virus co-evolution in HIV infection: paths for HIV vaccine development. Immunol. Rev. 275, 145-160 (2017).

5. Klein, F. et al. Somatic mutations of the immunoglobulin framework are generally required for broad and potent HIV-1 neutralization. Cell 153, 126-138 (2013).

6. Victora, G. D. \& Nussenzweig, M. C. Germinal centers. Annu. Rev. Immunol. 30, 429-457 (2012).

7. Schwickert, T. A. et al. A dynamic T cell-limited checkpoint regulates affinitydependent B cell entry into the germinal center. J. Exp. Med. 208, 1243-1252 (2011).

8. Escolano, A., Dosenovic, P. \& Nussenzweig, M. C. Progress toward active or passive HIV-1 vaccination. J. Exp. Med. 214, 3-16 (2017).

9. Escolano, A. et al. Sequential immunization elicits broadly neutralizing anti-HIV-1 Antibodies in Ig knockin mice. Cell 166, 1445-1458 (2016)

10. Tas, J. M. et al. Visualizing antibody affinity maturation in germinal centers. Science 351, 1048-1054 (2016).

11. Dal Porto, J. M., Haberman, A. M., Shlomchik, M. J. \& Kelsoe, G. Antigen drives very low affinity $B$ cells to become plasmacytes and enter germinal centers. $J$. Immunol. 161, 5373-5381 (1998).

12. Abbott, R. K. et al. Precursor frequency and affinity determine B cell competitive fitness in germinal centers, tested with germline-targeting HIV vaccine immunogens. Immunity 48, 133-146 (2018).

13. Dosenovic, P. et al. Anti-HIV-1 B cell responses are dependent on B cell precursor frequency and antigen-binding affinity. Proc. Natl Acad. Sci. USA 115 4743-4748 (2018)

14. Shih, T.-A. Y., Meffre, E., Roederer, M. \& Nussenzweig, M. C. Role of BCR affinity in T cell dependent antibody responses in vivo. Nat. Immunol. 3, 570-575 (2002).

15. Kong, L. et al. Supersite of immune vulnerability on the glycosylated face of HIV-1 envelope glycoprotein gp120. Nat. Struct. Mol. Biol. 20, 796-803 (2013).

16. Walker, L. M. et al. Broad neutralization coverage of HIV by multiple highly potent antibodies. Nature 477, 466-470 (2011).

17. Mouquet, $\mathrm{H}$. et al. Complex-type $N$-glycan recognition by potent broadly neutralizing HIV antibodies. Proc. Natl Acad. Sci. USA 109, E3268-E3277 (2012).

18. Freund, N. T. et al. Coexistence of potent HIV-1 broadly neutralizing antibodies and antibody-sensitive viruses in a viremic controller. Sci. Transl. Med. 9, eaal2144 (2017)

19. Sok, D. et al. A prominent site of antibody vulnerability on HIV envelope incorporates a motif associated with CCR5 binding and its camouflaging glycans. Immunity 45, 31-45 (2016).

20. Steichen, J. M. et al. HIV vaccine design to target germline precursors of glycan-dependent broadly neutralizing antibodies. Immunity $\mathbf{4 5}$, 483-496 (2016).

21. Sanders, R. W. et al. A next-generation cleaved, soluble HIV-1 Env trimer, BG505 SOSIP.664 gp140, expresses multiple epitopes for broadly neutralizing but not non-neutralizing antibodies. PLoS Pathog. 9, e1003618 (2013).

22. Gristick, H. B. et al. Natively glycosylated HIV-1 Env structure reveals new mode for antibody recognition of the CD4-binding site. Nat. Struct. Mol. Biol. 23 906-915 (2016).

23. Garces, F. et al. Structural evolution of glycan recognition by a family of potent HIV antibodies. Cell 159, 69-79 (2014).
24. Andrabi, R. et al. Glycans function as anchors for antibodies and help drive HIV broadly neutralizing antibody development. Immunity 47, 524-537 (2017).

25. Scharf, L. et al. Structural basis for germline antibody recognition of HIV-1 immunogens, eLife 5, e13783 (2016).

26. McCoy, L. E. et al. Holes in the glycan shield of the native HIV envelope are a target of trimer-elicited neutralizing antibodies. Cell Rep. 16, 2327-2338 (2016)

27. Duan, H. et al. Glycan masking focuses immune responses to the HIV-1 CD4-binding site and enhances elicitation of VRCO1-class precursor antibodies. Immunity 49, 301-311 (2018).

28. Garrity, R. R. et al. Refocusing neutralizing antibody response by targeted dampening of an immunodominant epitope. J. Immunol. 159, 279-289 (1997)

29. Klasse, P. J. et al. Epitopes for neutralizing antibodies induced by HIV-1 envelope glycoprotein BG505 SOSIP trimers in rabbits and macaques. PLoS Pathog. 14, e1006913 (2018).

30. Brune, K. D. et al. Plug-and-Display: decoration of virus-like particles via isopeptide bonds for modular immunization. Sci. Rep. 6, 19234 (2016)

31. Zakeri, B. et al. Peptide tag forming a rapid covalent bond to a protein, through engineering a bacterial adhesin. Proc. Natl Acad. Sci. USA 109, E690-E697 (2012).

32. Longo, N. S. et al. Multiple antibody lineages in one donor target the glycan-V3 supersite of the HIV-1 envelope glycoprotein and display a preference for quaternary binding. J. Virol. 90, 10574-10586 (2016).

33. Burton, D. R. \& Hangartner, L. Broadly neutralizing antibodies to HIV and their role in vaccine design. Annu. Rev. Immunol. 34, 635-659 (2016).

34. Sok, D. et al. The effects of somatic hypermutation on neutralization and binding in the PGT121 family of broadly neutralizing HIV antibodies. PLoS Pathog. 9 e1003754 (2013).

35. Lee, J. H., de Val, N., Lyumkis, D. \& Ward, A. B. Model building and refinement of a natively glycosylated HIV-1 Env protein by high-resolution cryoelectron microscopy. Structure 23, 1943-1951 (2015).

36. Zolla-Pazner, S. et al. Structure/function studies involving the V3 region of the HIV-1 envelope delineate multiple factors that affect neutralization sensitivity. $J$. Virol. 90, 636-649 (2015).

37. Murugan, R. et al. Clonal selection drives protective memory $B$ cell responses in controlled human malaria infection. Sci. Immunol. 3, eaap8029 (2018).

Acknowledgements We thank members of the Bjorkman, Martin and Nussenzweig laboratories for discussions, T. Eisenreich and S. Tittley for animal husbandry, K. Gordon for flow cytometry, S. Zolla-Pazner for providing the V3-consensus $\mathrm{C}$ peptide and anti-V3 monoclonal antibodies, M. Howarth for providing plasmids and advice for VLP expression and purification and A. Malyutin for help with cryo-EM data collection. Cryo-EM was done in the Beckman Institute Resource Center for Transmission Electron Microscopy at Caltech. This work was supported by the National Institute of Allergy and Infectious Diseases (NIAID) of the National Institutes of Health (NIH) Grant HIVRAD P01 Al100148 (to P.J.B. and M.C.N.), NIH Grant P50 GM082545-06 (to P.J.B.), NIH Center for HIV/AIDS Vaccine Immunology and Immunogen Discovery (CHAVI-ID) 1UM1 Al100663-01 (to M.C.N.), the Intramural Research Program of the NIAID (to M.A.M.), the National Center for Biomedical Glycomics P41GM103490 and NIGMS R01GM130915 (to L.W.), Gates CAVD grant OPP1146996 (to M.S.S. and D.C.M.), NIH/NIAID P01 Al138212 (to L.S., A.T.M. and M.C.N.) and the Robertson Fund of the Rockefeller University (M.C.N.). Additional support included an NSF GRFP (to M.E.A.), an EMBO fellowship (to J.M.), the HHMI Hanna Gray Fellowship and the Postdoctoral Enrichment Program from the Burroughs Welcome Fund (to C.O.B.). M.C.N. and D.J.I. are HHMI investigators.

Author contributions A.E., H.B.G., P.J.B. and M.C.N. designed the research. A.E. H.B.G., M.E.A., J.M., R.G., C.O.B., A.A.C., H.W., J.G., D.Y., J.R.K., Z.W., P.Z., L.N., K.H.Y., J.B., H.G., A.V.V. and M.S. performed the research. A.E., H.B.G., M.E.A., J.M., R.G., T.Y.O., J.P., A.P.W., P.J.B. and M.C.N. analysed the data. D.C.M. and M.S.S. supervised in vitro neutralization assays. A.G. supervised antibody production. M.A.M. planned and supervised the immunization experiments in macaques. D.J.I. planned and supervised adjuvant production. A.T.M. and L.S. produced anti-idiotypic antibodies. L.W. planned and supervised mass spectrometry experiments. A.E., H.B.G., M.E.A., P.J.B. and M.C.N. wrote the manuscript.

Competing interests There are patents on 3BNC117 and 10-1074 on which M.C.N. and P.J.B. are inventors. M.C.N. is a member of the Scientific Advisory Boards of Celldex and Frontier Biosciences.

\section{Additional information}

Extended data is available for this paper at https://doi.org/10.1038/s41586-

019-1250-z.

Supplementary information is available for this paper at https://doi.org/ 10.1038/s41586-019-1250-z.

Reprints and permissions information is available at http://www.nature.com/ reprints.

Correspondence and requests for materials should be addressed to P.J.B. or M.C.N.

Publisher's note: Springer Nature remains neutral with regard to jurisdictional claims in published maps and institutional affiliations.

(c) The Author(s), under exclusive licence to Springer Nature Limited 2019 


\section{METHODS}

Data reporting. No statistical methods were used to predetermine sample size. The experiments were not randomized and the investigators were not blinded to allocation during experiments and outcome assessment.

Envelope proteins. Env trimers were expressed as soluble native-like gp140 trimers $^{21}$. The newly engineered Env SOSIP trimers, RC1, RC1-4fill, RC1-Avitag, RC1-SpyTag, RC1-glycanKO, RC1-glycanKO-Avitag, RC1-glycanKO-GAIA and RC1-GAIA, BG505 ${ }^{21}$ and the BG505 variants $11 \mathrm{MUTB}, 10 \mathrm{MUT}, 7 \mathrm{MUT}$ and $5 \mathrm{MUT}^{20}$ were cloned in the pPPPI4 expression vector using synthetic gene fragments (Integrated DNA Technologies (IDT)). The glycan variants RC1 $\Delta 301$, RC1 $\Delta 332$ and 11 MUTB $\Delta 301$ were produced by site-directed mutagenesis (QuikChange Lightning Multi-site directed mutagenesis kit, Agilent Technologies). Specific modifications of each protein are listed in Extended Data Table 2.

Soluble Env trimers were expressed by transient transfection in HEK293-6E cells (National Research Council of Canada) or Expi293 cells (Life Technologies) and purified from cell supernatants by $2 \mathrm{G} 12$ or NIH45-46 immunoaffinity chromatography and size-exclusion chromatography (SEC) as previously described ${ }^{38}$. Proteins were stored at $4^{\circ} \mathrm{C}$ in $20 \mathrm{mM}$ Tris $\mathrm{pH} 8.0$ and $150 \mathrm{mM}$ sodium chloride (TBS buffer). SpyTagged immunogens were buffer-exchanged into $20 \mathrm{mM}$ sodium phosphate $\mathrm{pH} 7.5,150 \mathrm{mM} \mathrm{NaCl}$. Cell lines were not tested for mycoplasma contamination.

VLP production and conjugation. A C-terminal SpyTag sequence (13 residues) was added to RC1-4fill to form an irreversible isopeptide bond to SpyCatcher protein $^{31}$. We produced and purified SpyCatcher-AP20 $5^{39}$ VLPs as described ${ }^{30}$ and separated conjugated VLPs from free Env trimers by SEC on a Superdex 200 column. Conjugation of Env trimers was verified by negative-stain electron microscopy and/or SDS-PAGE (Fig. 3, Extended Data Fig. 4), and immunogen concentrations were estimated by comparing to known amounts of free immunogen run on the same SDS-PAGE gel. Conjugated and unconjugated VLPs were compared by negative-stain electron microscopy on a FEI Tecnai T12 transmission electron microscope at $120 \mathrm{keV}$ using a Gatan Ultrascan $2 \mathrm{k} \times 2 \mathrm{k}$ CCD detector. Mass spectrometry. The glycosylation profiles of RC1 and RC1-4fill were determined as previously described ${ }^{40}$. In brief, samples were denatured with Lys-C (Promega), Arg-C (Promega), Glu-C (Promega) and chymotrypsin (Promega). Following digestion, the samples were deglycosylated by Endo $\mathrm{H}$ (Promega) and PNGase F (Glyko, Prozyme) in the presence of ${ }^{18} \mathrm{O}$ water (Cambridge Isotope Laboratories). The resulting peptides were separated on an Acclaim PepMap RSLC C18 column $(75 \mu \mathrm{m} \times 15 \mathrm{~cm})$ and analysed using an Orbitrap Fusion Lumos Tribrid mass spectrometer (Thermo Fisher Scientific) with a 240-min linear gradient consisting of $1-100 \%$ solvent B over $180 \mathrm{~min}$ at a flow rate of $200 \mathrm{nl} \mathrm{min}^{-1}$. Full mass spectrometry scans were acquired using the Fusion instrument software (v.2.0, Thermo Fisher Scientific), and the resulting spectra were analysed and filtered using SEQUEST (Proteome Discoverer 1.4, Thermo Fisher Scientific) and ProteoIQ (v.2.7, Premier Biosoft). Site occupancy was calculated using spectral counts assigned to the ${ }^{18} \mathrm{O}$-Asp-containing (PNGase-F-cleaved) and/or HexNAc-modified (Endo-H-cleaved) peptides and their unmodified counterparts.

Animals. Mice carrying the inferred germline IgH and IgL human PGT121 and 10-1074 bNAbs (GL $\mathrm{GL}_{\mathrm{HL}} 121$ knock-in mice) were previously described ${ }^{9}$. Six-to-eight-week-old C57BL/6J male mice from The Jackson Laboratory were used for immunizations. All animal procedures were performed in accordance to protocols approved by the Rockefeller University Institutional Animal Care and Use Committee (IACUC). Male and female $\mathrm{GL}_{\mathrm{HL}} 121$ knock-in mice or C57BL/6J wild-type mice were equally distributed in groups and immunized intraperitoneally with $10 \mu \mathrm{g}$ of soluble SOSIP Env trimer in Ribi adjuvant (Sigma-Aldrich) (1:1)

Six-month-old New Zealand white rabbits (Covance) were used for immunizations. Rabbits were immunized subcutaneously with approximately $22 \mu \mathrm{g}$ of RC1-4fill SOSIP Env trimer conjugated to VLP (VLP-RC1-4fill) in an ISCOMs-like saponin adjuvant (see below). Serum samples were collected from mice and rabbits on weeks 0 and 2 after immunization. All procedures in rabbits were approved by the Denver PA IACUC.

Sixteen rhesus macaques (Macaca mulatta) of Indian genetic origin, two-to-four years of age, were housed and cared for in accordance with Guide for Care and Use of Laboratory Animals Report number NIH 82-53 (Department of Health and Human Services, Bethesda, 1985) in a biosafety level 2 NIH facility. All animal procedures and experiments were performed according to protocols approved by the IACUC of NIAID, NIH.

Macaques were immunized subcutaneously in the medial inner forelegs and hind legs (total of four sites per animal) with approximately $200 \mu \mathrm{g}$ (experiment 1; Fig. 3f) or $100 \mu \mathrm{g}$ (experiment 2; Extended Data Fig. 5a) of RC1-4fill SOSIP trimer conjugated to VLP (RC1-4fill VLP) adjuvated in IscoMPLA. Blood and lymph node biopsies were obtained from naive macaques and from the immunized macaques three weeks after immunization.
Adjuvant synthesis. ISCOM-like saponin adjuvant was prepared as described ${ }^{41}$. Final adjuvant concentrations were determined by cholesterol quantification (Sigma-Aldrich, MAK043).

ELISA. ELISAs with SOSIP Env trimers 11MUTB, RC1, 11MUTB $\Delta 301$, RC1 $\Delta 301, \mathrm{RC} 1-G A I A, R C 1$-glycanKO, RC1-glycanKO-GAIA, RC1 $\Delta 332$, BG505, 10MUT, 7MUT, 5MUT or the V3 loop-consensus C peptide (KGKGKGKGKGCTRPNNNTRKSIRIGPGQTFYATGDIIGDIRQAHC) were performed as described ${ }^{9}$. Serum samples were assayed at a 1:100 or 1:30 starting dilution and seven additional threefold serial dilutions. Mouse and human IgGs or human Fabs were evaluated at concentrations specified in the main text.

Alternatively, 96-well plates were directly coated with $50 \mu \mathrm{l}$ of a solution of Fab at $20 \mu \mathrm{g} \mathrm{ml}^{-1}$ in $1 \times \mathrm{PBS}$ overnight at $4^{\circ} \mathrm{C}$, washed and blocked as above and incubated in $50 \mu \mathrm{l}$ of a solution of RC1 or RC1-glycanKO-GAIA at $2 \mu \mathrm{g} \mathrm{ml}^{-1}$ in blocking buffer for $1 \mathrm{~h}$ at room temperature. Plates were washed as above and developed using a chimeric version (human Fabs and mouse crystallizable fragment $(\mathrm{Fc})$ ) of the $\mathrm{CD} 4$-binding site bNAb $3 \mathrm{BNC}^{42}$ at threefold serial dilutions starting at $5 \mu \mathrm{g} \mathrm{ml}^{-1}$ followed by anti-mouse IgG secondary antibody conjugated to HRP (Jackson ImmunoResearch, 115-035-071).

For anti-idiotype ELISAs, 96-well plates were coated with $50 \mu \mathrm{l}$ of a solution of IgG at $10 \mu \mathrm{g} \mathrm{ml}^{-1}$ in $1 \times$ PBS overnight at $4{ }^{\circ} \mathrm{C}$, washed and blocked as above and incubated with biotinylated anti-inferred germline PGT121 idiotypic antibody. Plates were developed with streptavidin conjugated to HRP.

Flow cytometry and single B cell sorting. Single-cell suspensions were obtained from the draining lymph nodes and spleens of immunized mice, and mature $B$ cells were isolated by negative selection using anti-CD43 magnetic beads (MACS) following the manufacturer's instructions.

Frozen peripheral blood mononuclear cells or cells from lymph node biopsies obtained from the naive and immunized macaques were thawed and washed in RPMI 1640 medium $(1 \times)$ (Gibco, 11875-093). Mouse or macaque cells were incubated with $100 \mu \mathrm{l}$ of FACS buffer $(1 \times$ PBS with $2 \%$ fetal bovine serum and $1 \mathrm{mM}$ EDTA) with mouse (BD Biosciences, 553142) or human (BD Biosciences, 564219) Fc Block, respectively, at a 1:500 dilution for $30 \mathrm{~min}$ on ice.

$\mathrm{RC1}$ and $\mathrm{RC1}$-glycanKO $\left(\mathrm{RC1}^{+} \mathrm{RC1}\right.$-glycan $\left.\mathrm{KO}^{-}\right)$tetramers were prepared by incubating $5 \mu \mathrm{g}$ of Avitagged and biotinylated RC1 (RC1-AviBio) or Avitagged and biotinylated RC1-glycanKO (RC1-glycanKO-AviBio) with fluorophore-conjugated streptavidin at a 1:200 dilution in $1 \times$ PBS for $30 \mathrm{~min}$ on ice.

$\mathrm{RC}^{+}{ }^{+} \mathrm{RC} 1$-glycanKO${ }^{-}$mouse $\mathrm{B}$ cells were isolated using $\mathrm{RC} 1-$ AviBio conjugated to streptavidin BV711 (BD Biosciences, 563262) and RC1-glycanKOAviBio conjugated to streptavidin-PE (BD Biosciences, 554061) as baits. $\mathrm{RC1}^{+} \mathrm{RC1}$-glycanKO $\mathrm{KO}^{-}$macaque $\mathrm{B}$ cells were isolated using $\mathrm{RC1}$-AviBio conjugated with streptavidin-PE and streptavidin-AF647 and RC1-glycanKO-AviBio conjugated with streptavidin-BV605 (BD Biosciences, 563260). Tetramers were mixed with the human or mouse antibody cocktails indicated below to a final concentration of $5 \mu \mathrm{g} \mathrm{ml}^{-1}$ each.

Mouse cells were stained with anti-CD4-APC-eFluor780 (Invitrogen, $47-$ 0042-82), anti-CD8-APC-eFluor780 (Invitrogen, 47-0081-82), anti-F4/80-APCeFluor780 (Invitrogen, 47-4801-82), anti-NK1.1-APC-eFluor780 (Invitrogen, 47-5941-82), anti-CD11b-APC-eFluor780 (eBioscience, 47-0112-82), antiCD11c-APC-eFluor780 (eBioscience, 47-0114-82), anti-Gr-1-APC-eFluor780 (Invitrogen, 47-5931-82), anti-B220-APC (Biolegend, 103212), anti-GL7-FITC (BD Biosciences, 553666) and anti-CD95-BV421 (BD Biosciences, 562633) antibodies at 1:200 dilution and the live/dead marker Zombie NIR (Biolegend, 77184 ) at a 1:400 dilution in FACS buffer. Macaque cells were stained with anti-CD16-APC-eFluor780 (Invitrogen, 47-0168-41), anti-CD8a-APC-eFluor780 (Invitrogen, 47-0086-42), anti-CD3-APC-eFluor780 (Invitrogen, 47-0037-41), anti-CD14-APC-eFluor780 (eBiosciences, 47-0149-41), anti-CD20-PE-Cy7 (BD, 335793), anti-CD38-FITC (Stem Cell Technologies, 60131FI), anti-IgG-BV421 (BD Biosciences, 562581) and anti-IgM-PerCP-Cy5.5 (BD Biosciences, 561285) antibodies at a 1:200 dilution and the live/dead marker Zombie NIR at a 1:400 dilution in FACS buffer.

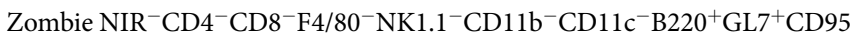
${ }^{+} \mathrm{RC1}^{+} \mathrm{RC1}$-glycanKO${ }^{-}$single cells were isolated from the mouse cell homogenates and Zombie $\mathrm{NIR}^{-} \mathrm{CD} 16^{-} \mathrm{CD}^{-}{ }^{-} \mathrm{CD} 3{ }^{-} \mathrm{CD} 14^{-} \mathrm{CD} 20^{+} \mathrm{CD}_{3} 8^{+} \mathrm{IgG}^{+/-}$double $\mathrm{RC1}^{+} \mathrm{RC1}$-glycan $\mathrm{KO}^{-}$single cells were isolated from the macaque cell homogenates using a FACS Aria III (Becton Dickinson).

Single cells were sorted into individual wells of a 96-well plate containing $5 \mu \mathrm{l}$ of lysis buffer (TCL buffer (Qiagen, 1031576) with 1\% of 2- $\beta$-mercaptoethanol). Plates were immediately frozen on dry ice and stored at $-80^{\circ} \mathrm{C}$.

Antibody sequencing and cloning. Single-cell RNA was purified using magnetic beads (RNAClean XP, A63987, Beckman Coulter). RNA was eluted from the mag-

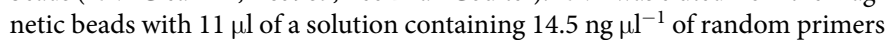
(Invitrogen, 48190-011), 0.5\% of tergitol (type NP-40, 70\% in $\mathrm{H}_{2} \mathrm{O}$, Sigma-Aldrich,

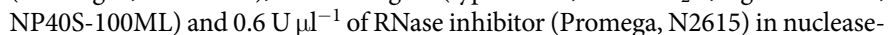
free water (Qiagen), and incubated at $65^{\circ} \mathrm{C}$ for $3 \mathrm{~min}$. cDNA was synthesized 
by reverse transcription (SuperScript III Reverse Transcriptase, Invitrogen, $18080-044,10,000 \mathrm{U})^{43}$. cDNA was stored at $-80^{\circ} \mathrm{C}$ or used for antibody gene amplification by nested polymerase chain reaction (PCR) after addition of $10 \mu \mathrm{l}$ of nuclease-free water.

Mouse and macaque antibody genes were cloned ${ }^{43}$ using the primers in Supplementary Table 6. PCR protocols were as follows for annealing $\left({ }^{\circ} \mathrm{C}\right) /$ elongation (s)/number of cycles: first PCR (IgG $\operatorname{IgH}$ and $\operatorname{Ig} \lambda$ ): 46/55/50; second PCR ( $\operatorname{IgG} \operatorname{IgH}$ and $\operatorname{Ig} \lambda$ ): 50/55/50.

Inferred germline macaque IgGs and Fabs were produced by reverting all nucleotide mutations in the $\mathrm{V}(\mathrm{D}) \mathrm{J}$ antibody genes to their corresponding inferred germline sequences while conserving the $\mathrm{N}$ nucleotides from the $\mathrm{V}(\mathrm{D}) \mathrm{J}$ junctions found in the mutated antibodies.

Antibody production and purification. Immunoglobulins were purified from $200 \mu \mathrm{l}$ of mouse or macaque serum using Ab Spin Trap Protein G Sepharose columns (GE Healthcare, 28-4083-47). Immunoglobulin-containing fractions were buffer-exchanged with PBS by overnight dialysis at $4^{\circ} \mathrm{C}$ (dialysis cassettes 20000 MWCO, Thermo Fisher Scientific, 66005).

For structural studies, mouse IgGs and macaque $\mathrm{His}_{6}$-tagged Fabs were expressed by transient transfection in HEK293-6E or Expi293 cells and purified from cell supernatants using protein A or G (GE Healthcare) (for IgGs) or Ni-NTA (GE Healthcare) or Ni Sepharose 6 Fast Flow (GE Healthcare) (for Fabs) chromatography and $\mathrm{SEC}^{44}$. Mouse Fab was obtained by digesting $\operatorname{IgG}$ at $1-5 \mathrm{mg} \mathrm{ml}^{-1}$ with ficin (Sigma-Aldrich). Fab was purified by protein G (GE Healthcare) and SEC chromatography ${ }^{45}$, followed by monoQ 5/50 (GE Healthcare) ion-exchange chromatography. The common inferred germline of the PGT121 and 10-1074 bNAbs ${ }^{17}$ was expressed as a $\mathrm{His}_{6}$-tagged Fab.

In vitro neutralization assay. TZM-bl assays were performed as previously described ${ }^{46}$. In brief, neutralization activity was calculated as a function of the reduction in Tat-induced luciferase expression in the TZM-bl reporter cell line after a single round of virus infection with Env pseudoviruses.

SPR and OCTET binding studies. SPR experiments were performed using a T200 (Biacore). For measuring the affinity for PGT121/10-1074 inferred germline Fab, protein A was immobilized on a CM 5 chip by primary amine chemistry (Biacore manual) and $200 \mathrm{nM} 8$ ANC195 ${ }_{\mathrm{G} 52 \mathrm{~K} 5}$ IgG or a non-HIV Env-binding IgG (mG053) was injected as previously described ${ }^{44}$. Then, $1 \mu \mathrm{M}$ human Fc was injected to block remaining protein A sites. After capturing $10 \mu \mathrm{M} \mathrm{RC1,11MUTB}$ or 10MUT, a concentration series of PGT121/10-1074 inferred germline Fab (fourfold dilutions from a top concentration of $160 \mu \mathrm{M}$ for 10MUT, and twofold dilutions from a top concentration of $150 \mu \mathrm{M}$ for $11 \mathrm{MUTB}$ and RC1) was injected, and binding reactions were allowed to reach equilibrium. $K_{\mathrm{D}}$ values were derived by nonlinear regression analysis of plots of $R_{\text {eq }}$ (equilibrium binding response) versus the log of the injected protein concentration and the data were fitted to a 1:1 binding model ${ }^{47}$. To measure the affinity of Ab275 MUR, a concentration series of Fab was injected over immobilized RC1 or 11MUTB (fourfold dilutions from a top concentration of $50 \mu \mathrm{M}) . K_{\mathrm{D}}$ values were calculated from the on/off rates $\left(k_{\mathrm{a}} / k_{\mathrm{d}}\right)$, which were derived using a 1:1 binding model from seven concentrations of Ab275 MUR Fab $(3.125 \mu \mathrm{M}$ to $0.763 \mathrm{nM})$. Flow cells were regenerated as previously described ${ }^{44}$ with $1 \mathrm{M}$ guanidine $\mathrm{HCl}$ and/or $10 \mathrm{mM}$ glycine $\mathrm{pH} 2.0$ at a flow rate of $90 \mu \mathrm{lmin}{ }^{-1}$.

OCTET experiments were performed using the OCTET Red96 system to determine affinities of inferred germline and mutated macaque Fabs for RC1. Biotinylated RC1-Avitag was immobilized on high-precision streptavidin (SAX) biosensors (FORTÉBIO) using a solution of biotinylated RC1-Avitag at $400 \mathrm{nM}$ in dilution buffer (FORTÉBIO). Four serial dilutions of each macaque Fab, one irrelevant Fab and 3BNC60 Fab were prepared in dilution buffer (FORTÉBIO). The binding experiment was performed at $30^{\circ} \mathrm{C}$ using the following protocol: baseline 1 (60 s), load RC1 (300 s), baseline 2 (200 s), Fab association (300 s) and Fab dissociation (600 s). Analysis was performed using OCTET software Data Analysis HT 10.0 (FORTÉBIO).

Cryo-EM sample and grid preparation. RC1 complexed with 10-1074 was prepared by incubating purified RC1 with 10-1074 Fab and a CD4-binding site (CD4bs) Fab at a 1:3:3 molar ratio (gp140 protomer:10-1074 Fab:CD4bs Fab) overnight at room temperature. The RC1-Fab complex was isolated by SEC in TBS (20 mM Tris pH 8.0, $100 \mathrm{mM} \mathrm{NaCl}$ ) using a Superdex-200 Increase 10/300 column (GE Healthcare). RC1-mouse/macaque Fab complexes were prepared by incubating purified RC1 with a mouse or macaque Fab and with $8 \mathrm{ANC} 195 \mathrm{Fab}^{42}$ at a 1:1.3:1.3 molar ratio as above and used without SEC purification. RC1-Fab complexes were diluted to $0.75-1.4 \mathrm{mg} \mathrm{ml}^{-1}$ in TBS, and $3 \mu \mathrm{l}$ was added to Quantifoil R1.2/1.3 300 mesh copper grids (Electron Microscopy Services) that had been freshly glow-discharged using a PELCO easiGlow (Ted Pella). Samples were vitrified in 100\% liquid ethane using a Mark IV Vitrobot (Thermo Fisher Scientific). Sample preparation conditions are summarized in Extended Data Table 1.

Cryo-EM data collection. RC1-Fab complexes were collected on a $200 \mathrm{kV}$ Thermo Fisher Talos Arctica electron microscope using EPU automated image acquisition software ${ }^{48}$. Movies were collected on a Falcon 3EC direct electron detector
(Thermo Fisher Scientific) operating in counting mode at a nominal magnification of $73,000 \times(1.436 \AA$ per pixel) using a defocus range of $-1.4 \mu \mathrm{m}$ to $-3.0 \mu \mathrm{m}$ or $-0.8 \mu \mathrm{m}$ to $-2.5 \mu \mathrm{m}$. Data for the RC1-10-1074 complex were collected across two separate sessions and combined during data processing. Microscope conditions are summarized in Extended Data Table 1.

Cryo-EM data processing. Movies were motion-corrected and dose-weighted using the MotionCor $2^{49}$ frame alignment program in RELION $-3^{50}$. Dose-weighted summed images were used for CTF determination using $\mathrm{Gct}^{51}$, and reference-free particle picking from each micrograph was achieved using Laplacian-of-Gaussian filtering in RELION $-3^{50}$. Unbinned extracted particles were imported into cryoSPARC v. $2^{52}$ and subjected to reference-free two-dimensional classification using a $240-\AA ̊$ circular mask. Particles from the best two-dimensional classes were selected for heterogeneous ab initio model generation (two models). The best model exhibited C3 symmetry and was used as an initial model for homogenous three-dimensional auto-refinement in cryoSPARC $v .2^{52}$. Resolutions were estimated using the gold standard Fourier shell correlation $(\mathrm{FSC}=0.143)^{53}$, and maps were auto-sharpened in cryoSPARC ${ }^{52}$. For interpreting $N$-linked glycans, maps were generated with overall $B$-factors ranging from -150 to $-400 \AA^{2}$ to improve local features and map connectivity ${ }^{54}$. See Extended Data Fig. 2 and Extended Data Table 1.

Model building. Initial coordinates were generated by docking reference models into the maps using UCSF Chimera ${ }^{55}$. For the RC1-10-1074 complex, BG505 Env and 10-1074 Fab (PDB code 53TZ) were docked into the density maps. For RC1 complexes with mouse or macaque Fabs, BG505 Env and PGT121/10-1074 inferred germline (PDB codes 5CEZ and 4FQQ) coordinates were docked into density maps. Initial models were refined into electron microscopy maps using rigid body refinement ${ }^{55}$. Models were built using $\mathrm{Fab}$ and $\mathrm{RC} 1$ sequences following iterative rounds of real-space refinement in Coot and PHENIX ${ }^{56,57}$. Coordinates for glycans were added as $\mathrm{Man}_{9}$ and then trimmed to fit the maps at $\sigma=5$. Model validation was done using MolProbity ${ }^{58}$ and Privateer ${ }^{59}$. Superposition calculations and molecular representations were generated with PyMOL (v.1.5.0.4 Schrodinger), UCSF Chimera ${ }^{55}$ and ResMap ${ }^{60}$

Analysis. MacVector v.15.5.3 was used for sequence analysis and graphs were created using $\mathrm{R}$ language. Flow cytometry data were processed using FlowJo v.10.5.0. GraphPad Prism 7 was used for data analysis. Immunoglobulin gene sequence $\mathrm{AB} 1$ files were converted to FASTQ format using the Biopython package. FASTQ files were trimmed by quality using cutadapt v.1.18 software. Igblast v.1.9.0 was used for VDJ assignment and clone analysis was performed using Change-O software v.0.3.7. For macaques, a custom VDJ database was created using previously reported immunoglobulin gene sequences ${ }^{61}$.

Quantification and statistical analysis. Statistical information, including $n$, mean and statistical significance values, is indicated in the text or the figure legends. GraphPad Prism 7 was used for statistical analysis by unpaired Student's $t$-test. Data were considered statistically significant at $* P \leq 0.05, * * P \leq 0.01$, $* * * P \leq 0.001$ and $* * * * P \leq 0.0001$

Reporting summary. Further information on research design is available in the Nature Research Reporting Summary linked to this paper.

\section{Data availability}

The atomic models and cryo-EM density maps generated during the current study have been deposited in the Protein Data Bank and Electron Microscopy Data Bank with accession numbers 6ORN and EMD-20175 (RC1-10-1074), 6ORQ and EMD-20178 (RC1-Ab275 $5_{\mathrm{MUR}}$ ), 6ORO and EMD-20176 (RC1-Ab874 $4_{\mathrm{NHP}}$ ), and 6ORP and EMD-20177 (RC1-Ab897 ${ }_{\mathrm{NHP}}$ ). Sequence datasets generated and analysed during the current study are available from the corresponding authors upon reasonable request.

38. Wang, H. et al. Asymmetric recognition of HIV-1 envelope trimer by V1V2 loop-targeting antibodies. eLife 6, e27389 (2017).

39. Tissot, A. C. et al. Versatile virus-like particle carrier for epitope based vaccines. PLOS ONE 5, e9809 (2010).

40. Duan, H. et al. Glycan masking focuses immune responses to the HIV-1 CD4-binding site and enhances elicitation of VRCO1-class precursor antibodies. Immunity 49, 301-311 (2018).

41. Lövgren-Bengtsson, K. \& Morein, B. in Vaccine Adjuvants: Preparation Methods and Research Protocols (ed. O'Hagan, D.) 239-258 (Humana, 2000).

42. Scheid, J. F. et al. Sequence and structural convergence of broad and potent HIV antibodies that mimic CD4 binding. Science 333, 1633-1637 (2011).

43. von Boehmer, L. et al. Sequencing and cloning of antigen-specific antibodies from mouse memory B cells. Nat. Protocols 11, 1908-1923 (2016).

44. Scharf, L. et al. Broadly neutralizing antibody 8 ANC195 recognizes closed and open states of HIV-1 Env. Cell 162, 1379-1390 (2015).

45. Diskin, R., Marcovecchio, P. M. \& Bjorkman, P. J. Structure of a clade C HIV-1 gp120 bound to CD4 and CD4-induced antibody reveals anti-CD4 polyreactivity. Nat. Struct. Mol. Biol. 17, 608-613 (2010).

46. Montefiori, D. C. Measuring HIV neutralization in a luciferase reporter gene assay. Methods Mol. Biol. 485, 395-405 (2009). 
47. Vaughn, D. E. \& Bjorkman, P. J. High-affinity binding of the neonatal Fc receptor to its IgG ligand requires receptor immobilization. Biochemistry 36, 9374-9380 (1997).

48. Tan, Y. Z Cheng, A., Potter, C. S. \& Carragher, B. Automated data collection in single particle electron microscopy. Microscopy 65, 43-56 (2016).

49. Zheng, S. Q. et al. MotionCor2: anisotropic correction of beam-induced motion for improved cryo-electron microscopy. Nat. Methods 14, 331-332 (2017).

50. Zivanov, J. et al. New tools for automated high-resolution cryo-EM structure determination in RELION-3. eLife 7, e42166 (2018)

51. Zhang, K. Gctf: real-time CTF determination and correction. J. Struct. Biol. 193 1-12 (2016).

52. Punjani, A., Rubinstein, J. L., Fleet, D. J. \& Brubaker, M. A. cryoSPARC: algorithms for rapid unsupervised cryo-EM structure determination. Nat. Methods 14 290-296 (2017).

53. Scheres, S. H. \& Chen, S. Prevention of overfitting in cryo-EM structure determination. Nat. Methods 9, 853-854 (2012).
54. Terwilliger, T. C., Sobolev, O. V., Afonine, P. V. \& Adams, P. D. Automated map sharpening by maximization of detail and connectivity. Acta Crystallogr. 74, 545-559 (2018).

55. Goddard, T. D., Huang, C. C. \& Ferrin, T. E. Visualizing density maps with UCSF Chimera. J. Struct. Biol. 157, 281-287 (2007).

56. Adams, P. D. et al. PHENIX: a comprehensive Python-based system for macromolecular structure solution. Acta Crystallogr. D 66, 213-221 (2010).

57. Emsley, P., Lohkamp, B., Scott, W. G. \& Cowtan, K. Features and development of Coot. Acta Crystallogr. D 66, 486-501 (2010).

58. Chen, V. B. et al. MolProbity: all-atom structure validation for macromolecular crystallography. Acta Crystallogr. D 66, 12-21 (2010).

59. Agirre, J. et al. Privateer: software for the conformational validation of carbohydrate structures. Nat. Struct. Mol. Biol. 22, 833-834 (2015)

60. Kucukelbir, A., Sigworth, F. J. \& Tagare, H. D. Quantifying the local resolution of cryo-EM density maps. Nat. Methods 11, 63-65 (2014).

61. Corcoran, M. M. et al. Production of individualized V gene databases reveals high levels of immunoglobulin genetic diversity. Nat. Commun. 7, 13642 (2016) 


\begin{tabular}{|c|c|c|c|}
\hline \multirow{2}{*}{} & \multicolumn{3}{|c|}{ IC50 values $(\mu \mathrm{g} / \mathrm{mL})$} \\
\cline { 2 - 4 } & $10-1074$ & PGT121 & BG1 \\
\hline & $+N 332$ & $+N 332$ & $+N 160$ \\
\hline$+N 156$ & $0.20(n=75)$ & $0.14(n=170)$ & $5.80(n=102)$ \\
\hline$-N 156$ & $0.05(n=8)$ & $0.03(n=9)$ & $30.0(n=8)$ \\
\hline$+N 301$ & $0.16(n=81)$ & $0.11(n=173)$ & - \\
\hline$-N 301$ & $1.05(n=2)$ & $15.91(n=6)$ & - \\
\hline$+N 137$ & $0.04(n=11)$ & $0.10(n=34)$ & - \\
\hline$-N 137$ & $0.21(n=72)$ & $0.14(n=145)$ & - \\
\hline
\end{tabular}

b
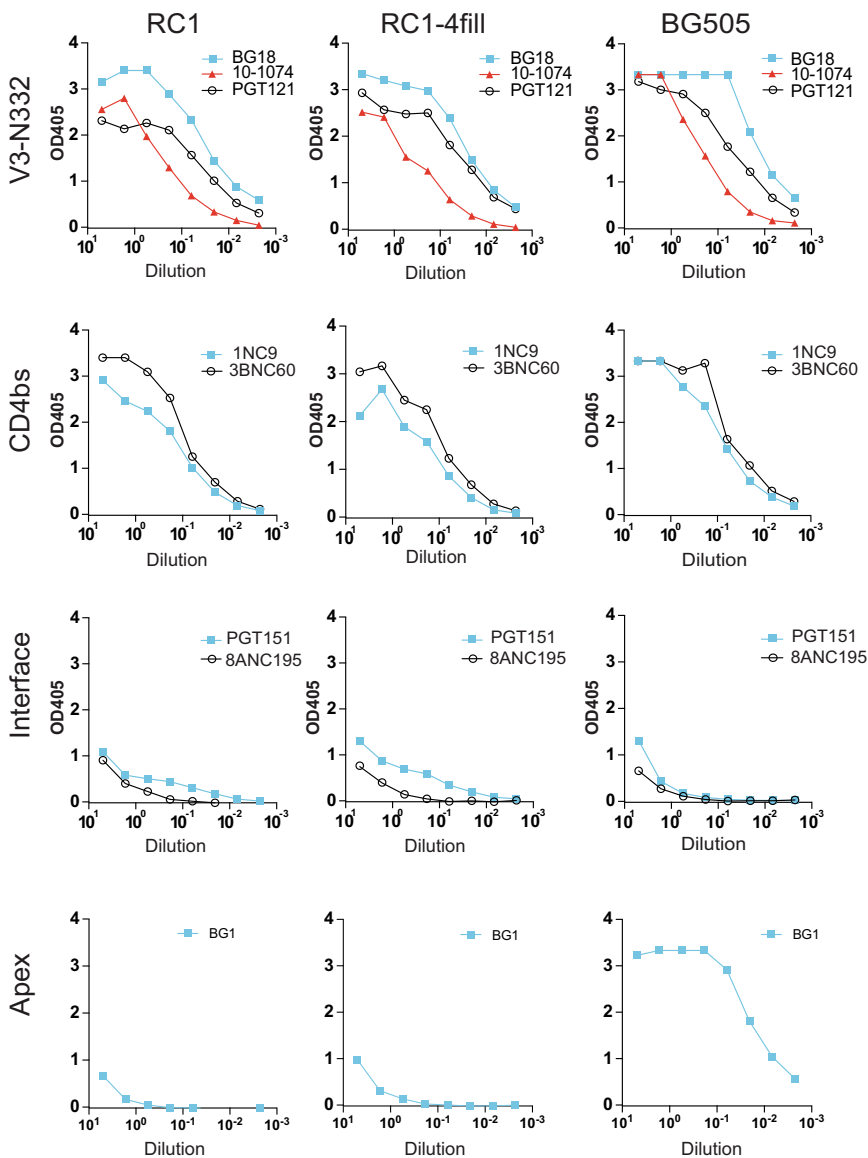

Extended Data Fig. 1 | RC1 characterization. a, Comparison of geometric mean half-maximum inhibitory concentrations $\left(\mathrm{IC}_{50}\right.$ ) for V3-glycan patch bNAbs (10-1074 and PGT121) and an N156 glycan-dependent V1V2 bNAb (BG1) evaluated against HIV-1 strains that either contained or did not contain a PNGS at the indicated positions (the number of HIV-1 strains is indicated in the parentheses). Values of $\mathrm{IC}_{50}$ greater than $50 \mu \mathrm{g} \mathrm{ml}^{-1}$ were set to $50 \mu \mathrm{g} \mathrm{ml}^{-1}$ for geometric mean calculations. Whereas V3-glycan patch bNAbs showed enhanced neutralization upon removal of the N156 glycan, removal of nearby glycans (N137 or N301) diminished or had little effect on neutralization. b, ELISA data showing the binding of different classes of bNAbs to RC1, RC1-4fill and BG505. bNAbs were evaluated at $5 \mu \mathrm{g} \mathrm{ml}^{-1}$ and seven additional threefold dilutions. $n=2$. RC1 and RC1-4fill show similar binding patterns for V3-glycan patch bNAbs, CD4-binding site bNAbs (CD4bs) and gp120-gp41 interface bNAbs, but reduced binding to BG1, a V1V2 bNAb that interacts with the N156 glycan (see a). 
a $10-1074-\mathrm{RC} 1$

Micrograph
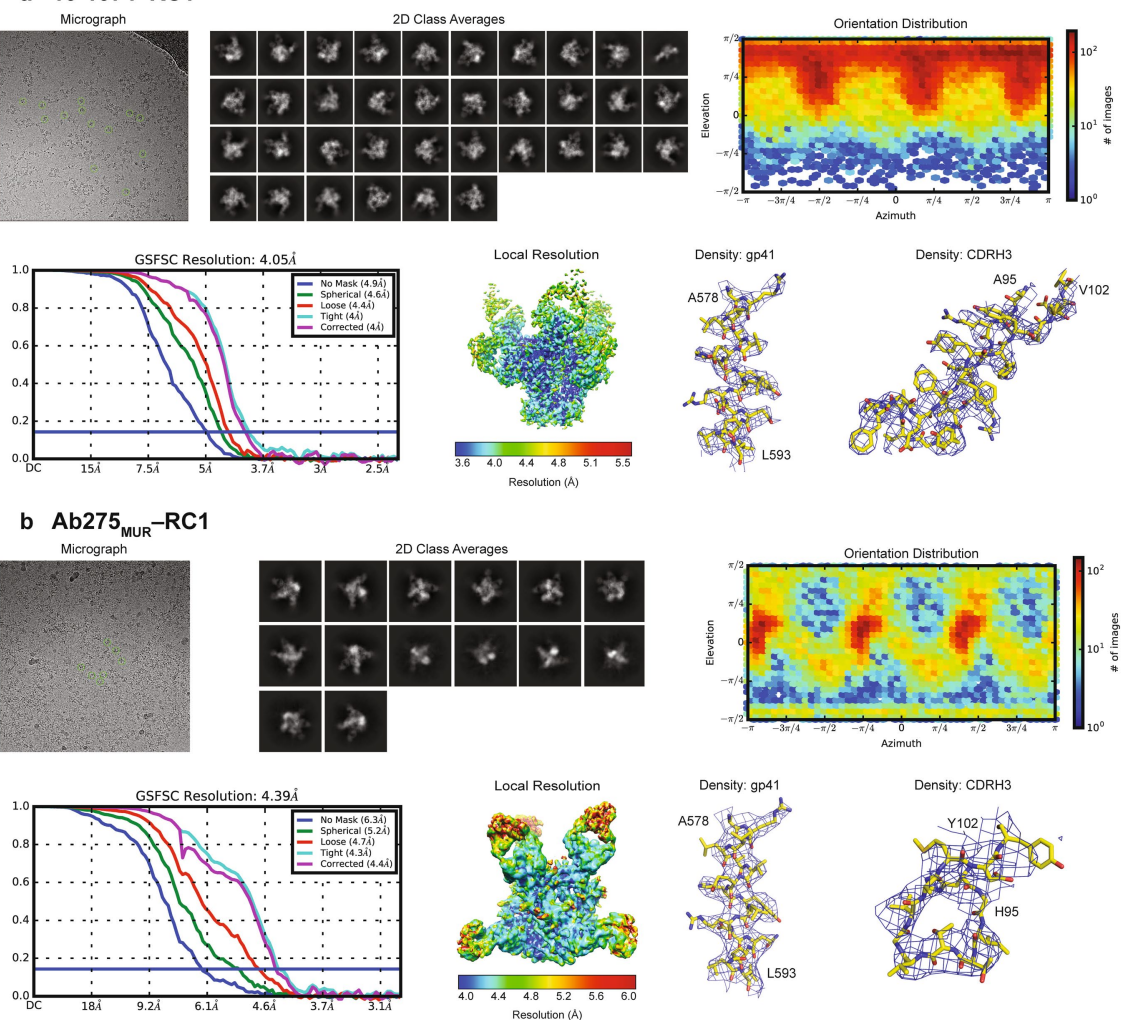

C Ab874 $_{\text {NHP }}-R C 1$
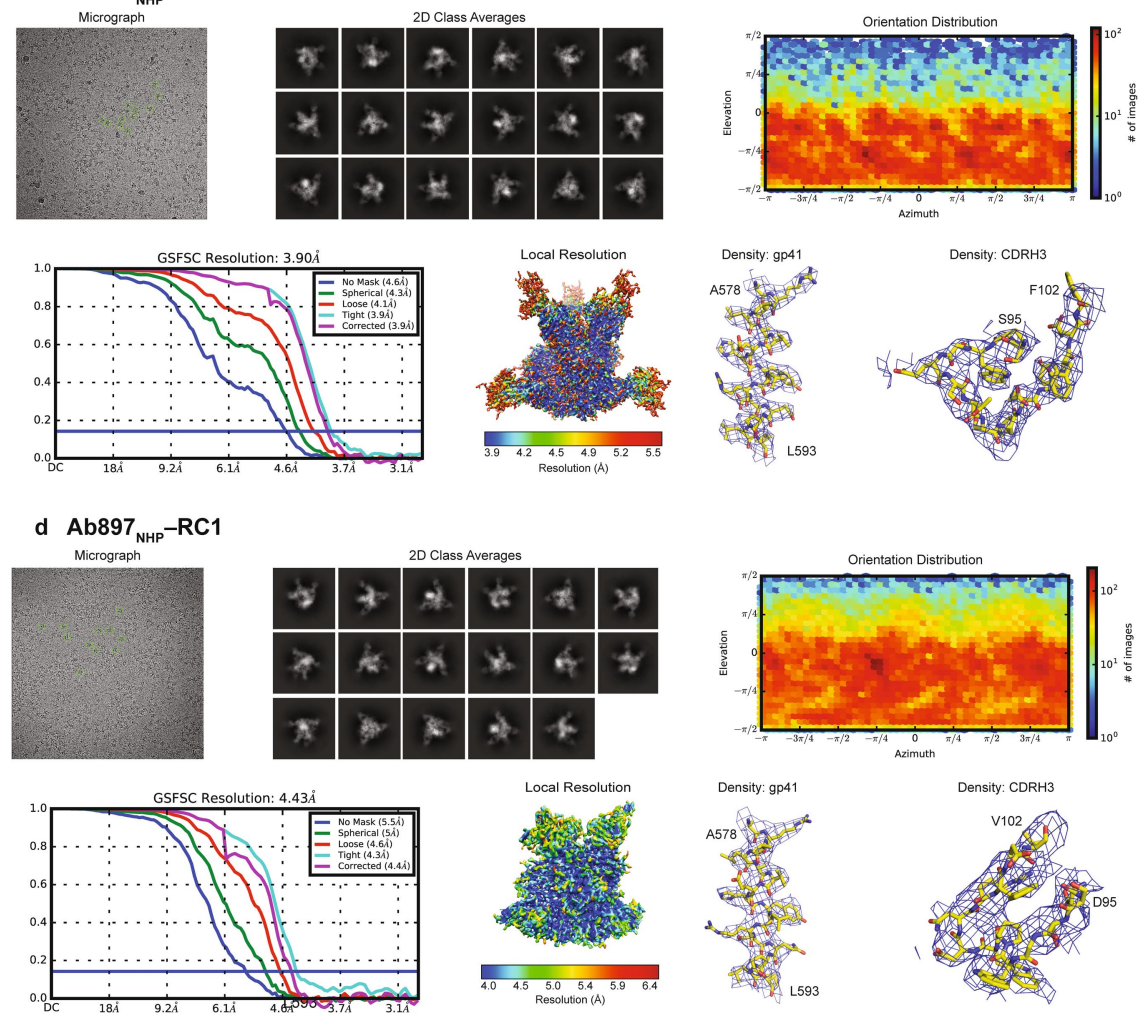

Extended Data Fig. 2 | Cryo-EM data collection and processing for RC1 complexes. a-d, A representative micrograph, selected two-dimensional class averages, orientation distribution summary, GSFSC resolution plot, local resolution (calculated using ResMap) and representative density maps contoured at $7 \sigma$ for a gp41 helix and antibody CDRH3 are shown. a, The $10-1074-\mathrm{RC} 1$ complex. b, The Ab275 ${ }_{\mathrm{MUR}}-\mathrm{RC} 1$ complex. $\mathbf{c}$, The Ab874 ${ }_{\mathrm{NHP}}-\mathrm{RC} 1$ complex. d, The Ab897 ${ }_{\mathrm{NHP}}-\mathrm{RC} 1$ complex. 
a
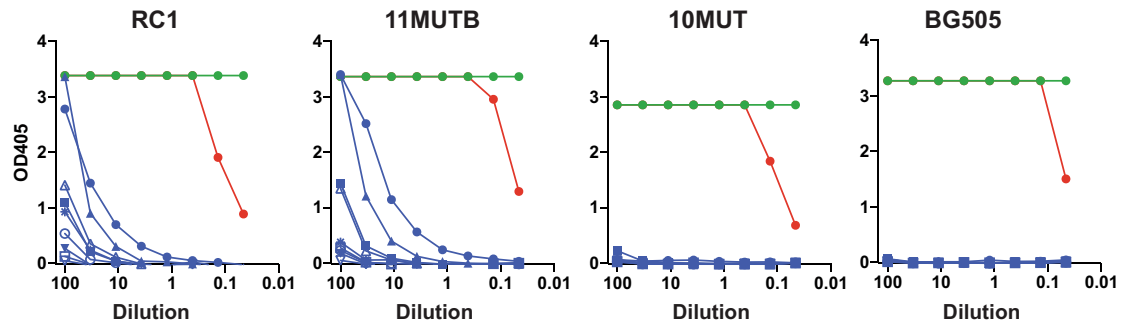

- Imm. mouse serum

$$
\begin{aligned}
& \rightarrow \text { 10-1074 } \\
& \rightarrow \text { 3BNC117 }
\end{aligned}
$$

b
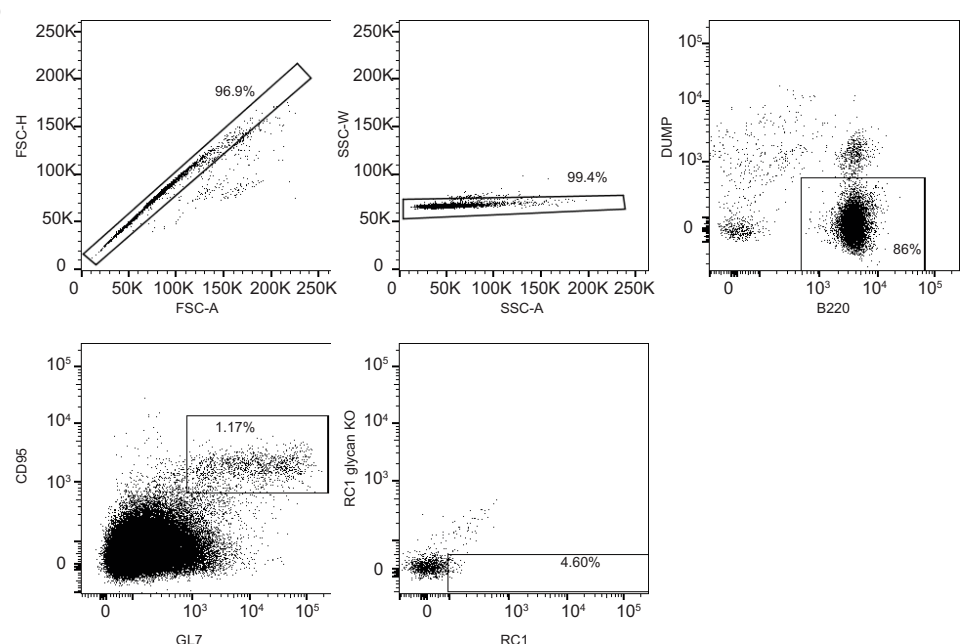

d
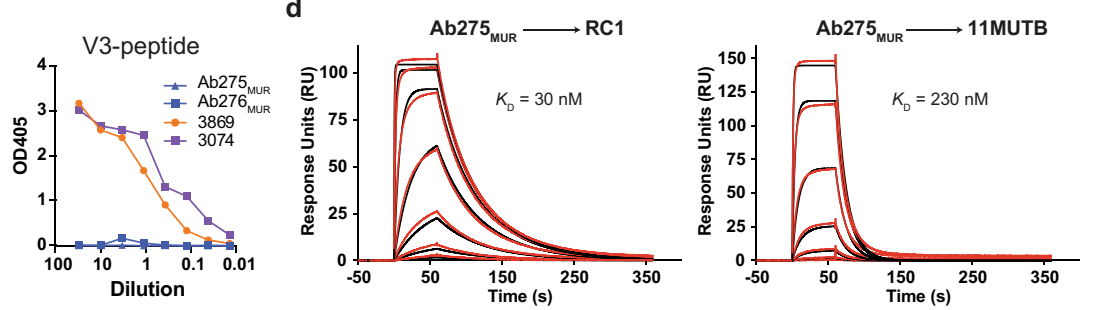

$\mathrm{RC} 1$
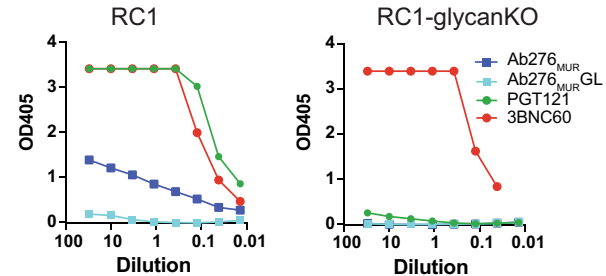

Extended Data Fig. $3 \mid$ Antibody responses in wild-type mice. a, ELISA cross-reactivity of serum from RC1-immunized wild-type mice to 11MUTB. Binding of the serum from wild-type mice primed with RC1 to RC1, 11MUTB, 10MUT and BG505 is shown in blue. Binding of the human bNAbs 10-1074 (green) and 3BNC117 (red) was evaluated at $5 \mu \mathrm{g} \mathrm{ml}^{-1}$ as a control. $n=2$. b, FACS plots showing the gating strategy to isolate single $\mathrm{RC} 1^{+} \mathrm{RC} 1$-glycan $\mathrm{KO}^{-}$germinal centre $\mathrm{B}$ cells

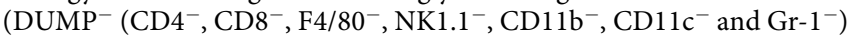
$\mathrm{B} 220^{+} \mathrm{CD}^{-} 5^{+} \mathrm{GL}^{+} \mathrm{RC} 1$-glycanKO ${ }^{-} \mathrm{RC}^{+}$) from the spleen and draining lymph nodes of wild-type mice primed with RC1 or RC1-4fill. c, Binding of the mouse antibodies Ab275 $5_{\text {MUR }}$ and Ab276 $6_{\text {MUR }}$ to a V3 loop-consensus C peptide (see Methods). Human antibodies 3869 and 3074 were used as positive controls. Antibodies were evaluated at $30 \mu \mathrm{g} \mathrm{ml}^{-1} . n=2$. d, Representative sensograms from two independent SPR-binding experiments of Ab275 $5_{\text {MUR }}$ Fab injected over immobilized RC1 (left) or 11MUTB (right). Experimental binding curves (red) are overlaid with predicted curves (black) derived from a 1:1 binding model. Representative of 3 independent experiments. e, Binding of Ab276 $6_{M U R}$ and its inferred germline version (Ab276 $\left.{ }_{\mathrm{MUR}} \mathrm{GL}\right)$ to RC1 (left) and RC1glycanKO (right) at $30 \mu \mathrm{g} \mathrm{ml}^{-1}$. The human monoclonal antibodies PGT121 (green) and 3BNC60 (red) were used as controls at $5 \mu \mathrm{g} \mathrm{ml}^{-1}$. 

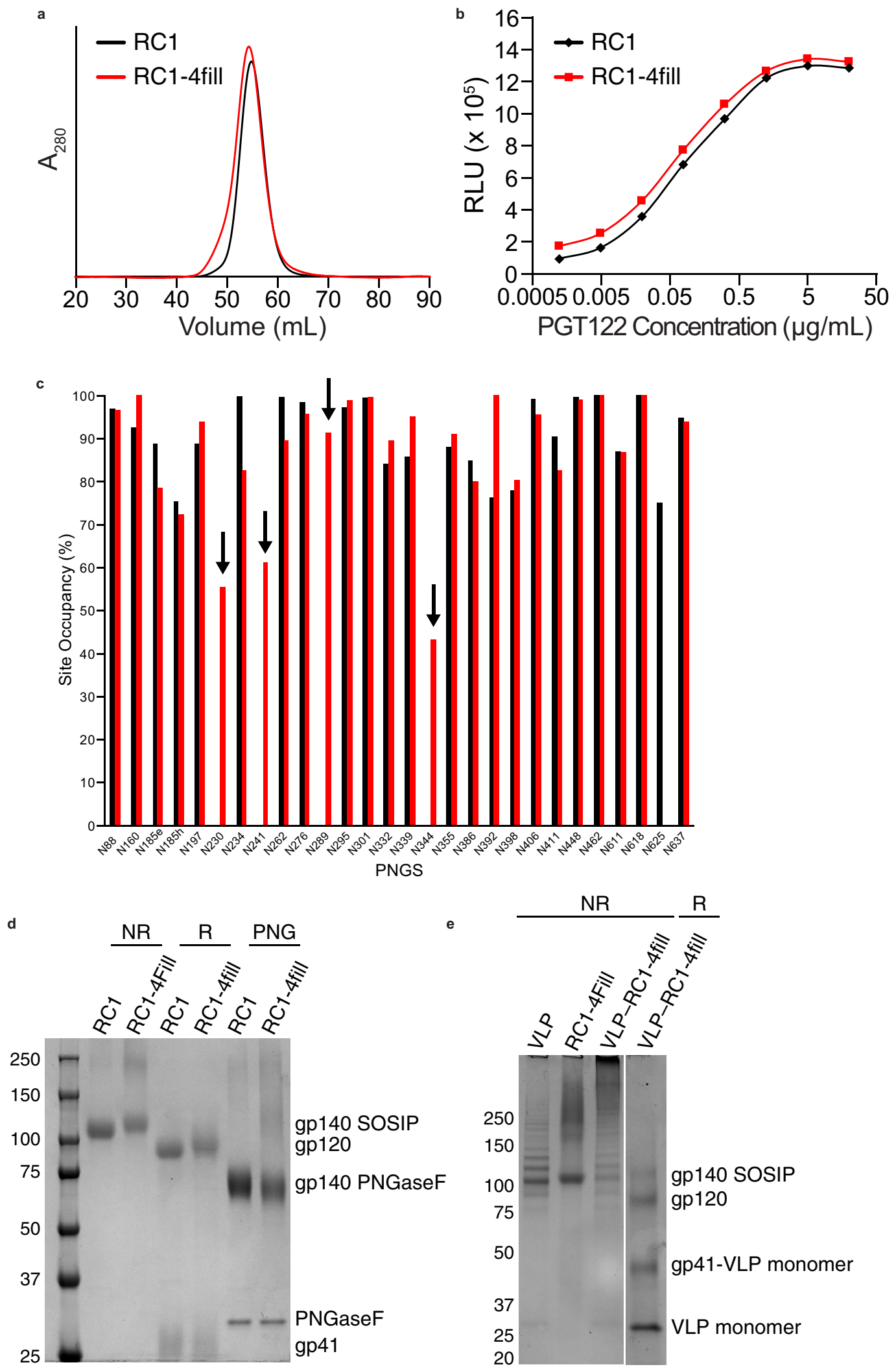

Extended Data Fig. 4 | Characterization of RC1, RC1-4fill and VLPs. a, SEC profiles of RC1 and RC1-4fill showing a larger apparent hydrodynamic radius for RC1-4fill compared with RC1, consistent with addition of extra glycans at the introduced PNGSs. b, ELISAs showing comparable binding of PGT122 Fab to RC1 and RC1-4fill. RLU, relative luminescence unit. c, Glycan site occupancy for each PNGS in RC1 and
RC1-4fill determined by mass spectrometry. d, SDS-PAGE analysis for $\mathrm{RC} 1$ and RC1-4fill under non-reducing (NR), reducing (R) and PNGaseFtreated (PNG) conditions. e, SDS-PAGE analysis for VLP, SpyTagged RC1-4fill and VLP-RC1-4fill under non-reducing and reducing conditions. 
a

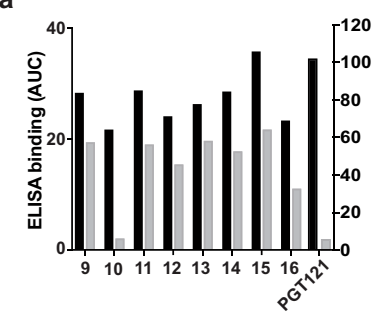

d

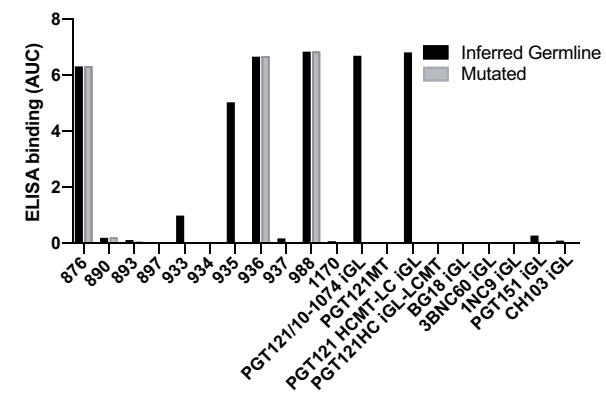

b

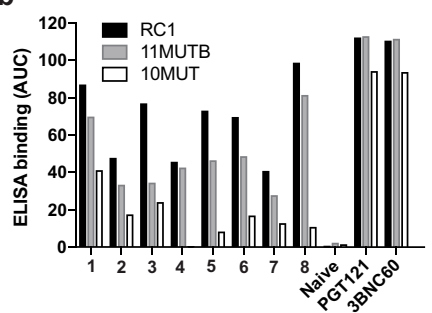

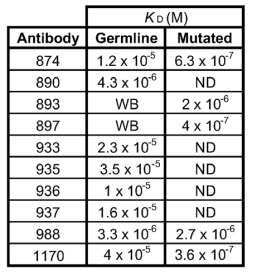

f

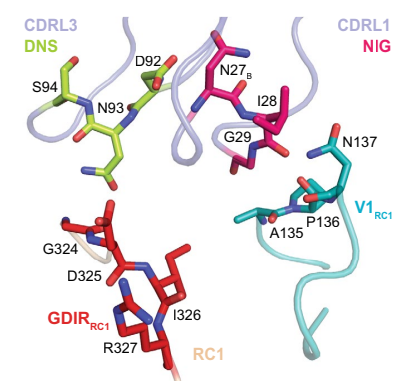

e
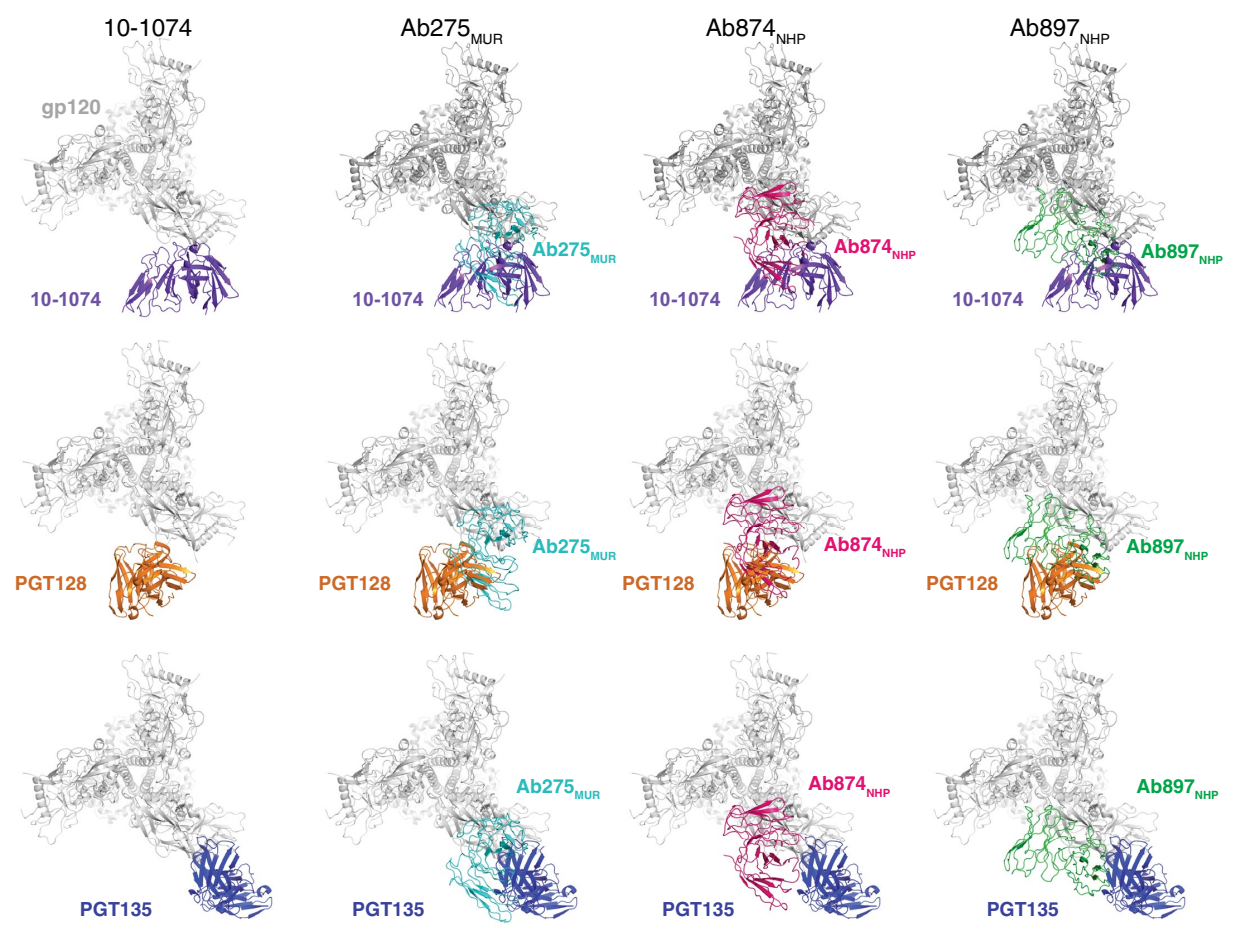

Extended Data Fig. 5 | Characterization of antibody responses in macaques. a, Binding of serum from macaques primed with RC1-4fill VLPs. ELISAs of the serum from eight macaques primed with RC1-4fill VLPs and PGT121 to RC1 (black) and RC1-glycanKO (grey) are shown. b, Binding of serum from macaques primed with RC1-4fill VLPs. ELISA of the serum from eight macaques primed with RC1-4fill VLP and one naive macaque to RC1 (black) and the sequentially less modified Env proteins 11MUTB (grey) and 10MUT (white). The human bNAbs PGT121 and 3BNC60 were used as controls at $5 \mu \mathrm{g} \mathrm{ml}^{-1}$, and the serum was evaluated at a 1:100 dilution and seven additional threefold serial dilutions. c, The affinities $\left(K_{\mathrm{D}}\right)$ for $\mathrm{RC} 1$ of different macaque antibodies isolated after a prime with VLP-RC1-4fill and the corresponding inferred germline-reverted antibodies as determined by bio-layer interferometry (OCTET). d, Binding of an anti-idiotypic antibody that recognizes the inferred germline of PGT121/10-1074 to monoclonal antibodies isolated from macaques primed with VLP-RC1-4fill. The inferred germline (iGL) of PGT121/10-1074, two chimeric antibodies comprising the mutated (MT) heavy chain (HC) and inferred germline light chain (LC) of PGT121 (PGT121 HCMT-LC iGL) or the inferred germline HC and the mutated LC of PGT121 (PGT121HC iGL-LCMT) and different inferred germline bNAbs were used as controls. a, b, d, Results are shown as the area under the ELISA curve (AUC). e, Comparison of binding mode between the vaccine-elicited antibodies (Ab275 $5_{\mathrm{MUR}}, \mathrm{Ab} 874_{\mathrm{NHP}}$ and $\mathrm{Ab} 897_{\mathrm{NHP}}$ ) and the V3-glycan patch bNAbs 10-1074, PGT128 and PGT135. RC1 trimer is shown in grey from above and all Fabs are modelled onto the same trimer. For clarity, only one Fab per trimer is shown. f, Interactions between Ab897 ${ }_{\mathrm{NHP}}$ conserved light chain motifs and RC1 gp120. Lime, DNS motif in CDRL3; red, gp120 GDIR; pink, NIG motif in CDRL1; teal, gp120 V1 loop. Each AUC value corresponds to one ELISA curve. 
Extended Data Table 1 | Cryo-EM data collection, refinement and validation statistics

\begin{tabular}{|c|c|c|c|c|}
\hline & $\begin{array}{l}\mathrm{RC} 1-10-1074 \\
(\mathrm{EMDB}-20175) \\
(\mathrm{PDB} 6 \mathrm{ORN})\end{array}$ & $\begin{array}{l}\mathrm{RC} 1-\mathrm{Ab} 275_{\mathrm{MUR}} \\
\text { (EMDB-20178) } \\
\text { (PDB 6ORQ) }\end{array}$ & $\begin{array}{l}\mathrm{RC} 1-\mathrm{Ab} 874_{\mathrm{NHP}} \\
(\mathrm{EMDB}-20176) \\
(\mathrm{PDB} 6 \mathrm{ORO})\end{array}$ & $\begin{array}{l}\mathrm{RC} 1-\mathrm{Ab} 897_{\mathrm{NHP}} \\
(\mathrm{EMDB}-20177) \\
\text { (PDB 6ORP) }\end{array}$ \\
\hline \multicolumn{5}{|l|}{ Data collection and processing } \\
\hline Magnification & $73,000 \mathrm{x}$ & $73,000 \mathrm{x}$ & $73,000 \mathrm{x}$ & $73,000 \mathrm{x}$ \\
\hline Voltage $(\mathrm{kV})$ & 200 & 200 & 200 & 200 \\
\hline Electron exposure $\left(\mathrm{e}-/ \AA^{2}\right)$ & 39.1 & 40 & 40 & 40 \\
\hline Defocus range $(\mu \mathrm{m})$ & $1-3.4$ & $0.8-2.5$ & $0.8-2.5$ & $0.8-2.5$ \\
\hline Pixel size $(\AA)$ & 1.436 & 1.436 & 1.436 & 1.436 \\
\hline Symmetry imposed & C3 & C3 & C3 & C3 \\
\hline Initial particle images (no.) & 145,907 & 172,558 & 188,004 & 314,471 \\
\hline Final particle images (no.) & 122,013 & 49,308 & 86,564 & 158,954 \\
\hline $\begin{array}{l}\text { Map resolution }(\AA) \\
\text { FSC threshold - } 0.143\end{array}$ & 4.05 & 4.39 & 3.90 & 4.43 \\
\hline Map resolution range $(\AA)$ & $3.6-6$ & $4.2-7$ & $3.5-6.5$ & $4.2-7$ \\
\hline \multicolumn{5}{|l|}{ Refinement } \\
\hline Initial model used (PDB code) & ab initio & ab initio & ab initio & ab initio \\
\hline Model resolution $(\AA)$ & 4.0 & 4.4 & 3.9 & 4.4 \\
\hline FSC threshold & 0.143 & 0.143 & 0.143 & 0.143 \\
\hline Model resolution range $(\AA)$ & $3.8-4.2$ & $3.8-4.4$ & $3.6-4.0$ & $4.0-4.5$ \\
\hline Map sharpening $B$ factor $\left(\AA^{2}\right)$ & -192.6 & -252.4 & -230.0 & -322.1 \\
\hline \multicolumn{5}{|l|}{ Model composition } \\
\hline Non-hydrogen atoms & 20,475 & 19,932 & 20,010 & 19,590 \\
\hline Protein residues & 2,418 & 2,430 & 2,421 & 2,397 \\
\hline \multirow[t]{2}{*}{ Ligands } & BMA:9 NAG:81 & BMA:6 NAG:54 & BMA:9 NAG:66 & BMA:6 NAG:48 \\
\hline & MAN:21 & MAN:6 & MAN: 12 & MAN:18 \\
\hline \multicolumn{5}{|l|}{$B$ factors $\left(\AA^{2}\right)$} \\
\hline Protein & 52.4 & 162.6 & 74.6 & 135.0 \\
\hline Ligand & 73.5 & 199.3 & 100.2 & 188.2 \\
\hline \multicolumn{5}{|l|}{ R.m.s. deviations } \\
\hline Bond lengths $(\AA)$ & 0.007 & 0.007 & 0.007 & 0.008 \\
\hline Bond angles $\left(^{\circ}\right)$ & 0.884 & 1.108 & 1.091 & 1.084 \\
\hline \multicolumn{5}{|l|}{ Validation } \\
\hline MolProbity score & 2.07 & 2.19 & 1.92 & 2.43 \\
\hline Clashscore & 10.87 & 8.12 & 4.67 & 7.61 \\
\hline Poor rotamers $(\%)$ & 0.95 & 1.28 & 1.29 & 2.87 \\
\hline \multicolumn{5}{|l|}{ Ramachandran plot } \\
\hline Favored $(\%)$ & 91.0 & 84.8 & 88.0 & 84.9 \\
\hline Allowed $(\%)$ & 8.9 & 15.0 & 12.0 & 15.1 \\
\hline Disallowed (\%) & 0.1 & 0.1 & 0 & 0 \\
\hline
\end{tabular}


Extended Data Table 2 | HIV-1 Env-based proteins

\begin{tabular}{|c|c|c|c|}
\hline \multirow{2}{*}{ Protein } & \multicolumn{2}{|c|}{ PNGS } & \multirow{2}{*}{ Other modifications } \\
\hline & Deleted & Added & \\
\hline RC1 & $133,137,156$ & - & V134Y, T135A, I138L, T139L, D140S, D141N, T320F, Q328M, T415V, MD39* \\
\hline RC1-4FILL & $133,137,156$ & $230,241,289,344$ & V134Y, T135A, I138L, T139L, D140S, D141N, T320F, Q328M, T415V, MD39* \\
\hline RC1-glycanKO & $133,137,156,301,332$ & - & V134Y, T135A, I138L, T139L, D140S, D141N, T320F, Q328M, T415V, H330A, MD39* \\
\hline RC1-glycanKO-GAIA & $133,137,156,301,332$ & - & V134Y, T135A, I138L, T139L, D140S, D141N, T320F, Q328M, T415V, GDIR /GAIA, H330A, MD39* \\
\hline RC1-GAIA & $133,137,156$ & - & V134Y, T135A, I138L, T139L, D140S, D141N, T320F, Q328M, T415V, GDIR /GAIA, MD39* \\
\hline $11 \mathrm{MUTB} \Delta 301$ & $133,137,301$ & - & V134Y, T135A, I138L, T139L, D140S, D141N, T320F, Q328M, T415V, MD39* \\
\hline $\mathrm{RC} 1 \Delta 301$ & $133,137,156,301$ & - & V134Y, T135A, I138L, T139L, D140S, D141N, T320F, Q328M, T415V, MD39* \\
\hline $\mathrm{RC} 1 \Delta 332$ & $133,137,156,332$ & & V134Y, T135A, I138L, T139L, D140S, D141N, T320F, Q328M, T415V, MD39* \\
\hline 11MUTB & 133,137 & - & V134Y, T135A, I138L, T139L, D140S, D141N, T320F, Q328M, T415V, MD39* \\
\hline 10MUT & 133,137 & - & V134Y, T135A, N136P, N137F, I138L, T139I, D140N, T320F, Q328M, MD39* \\
\hline 7MUT & 133,137 & - & V134Y, T135A, N136P, N137F, I138L, T139I, D140N, MD39* \\
\hline 5MUT & - & - & V134Y, N136P, I138L, D140N, MD39* \\
\hline BG505 & - & - & MD39* \\
\hline RC1-4fill VLP & $133,137,156$ & $230,241,289,344$ & V134Y, T135A, I138L, T139L, D140S, D141N, T320F, Q328M, T415V, Spytag, MD39* \\
\hline RC1-Avitag & $133,137,156$ & - & V134Y, T135A, I138L, T139L, D140S, D141N, T320F, Q328M, T415V, Avitag, MD39* \\
\hline RC1-glycanKO-Avitag & $133,137,156,301,332$ & - & V134Y, T135A, I138L, T139L, D140S, D141N, T320F, Q328M, T415V, H330A, Avitag, MD39* \\
\hline
\end{tabular}

The modifications of all Env proteins used in this study. Asterisk refers to MD39 mutations: T106E, M271I, F288L, N363Q, R304V, A319Y, F519S, A561P, L568D, V570H, R585H. 


\section{RESEARCH ARTICLE}

Extended Data Table 3 | Results of neutralization assays in TZM-bl cells

\begin{tabular}{|c|c|c|}
\hline \multirow[b]{2}{*}{ Sample } & \multicolumn{2}{|c|}{ ID50 in TZM-bl cells } \\
\hline & BG505 $\Delta \mathrm{CT} / \mathrm{T} 332 \mathrm{~N}$ & Du156.12 \\
\hline NHP 1 & $<25$ & $<25$ \\
\hline NHP 2 & $<25$ & $<25$ \\
\hline NHP 3 & $<25$ & $<25$ \\
\hline NHP 4 & $<25$ & $<25$ \\
\hline NHP 5 & $<25$ & $<25$ \\
\hline NHP 6 & $<25$ & $<25$ \\
\hline NHP 7 & $<25$ & $<25$ \\
\hline NHP 8 & $<25$ & $<25$ \\
\hline NAÏVE NHP & $<25$ & $<25$ \\
\hline PGT121 & 0.03 & $<0.02$ \\
\hline
\end{tabular}

b

\begin{tabular}{|c|c|c|c|c|c|c|c|c|c|}
\hline \multirow[b]{3}{*}{ Virus ID } & \multirow{2}{*}{\multicolumn{9}{|c|}{ ID50 in TZM-bl cells }} \\
\hline & & & & & & & & & \\
\hline & NHP 1 & NHP 2 & NHP 3 & NHP 4 & NHP 5 & NHP 6 & NHP 7 & NHP 8 & NAÏVE NHP \\
\hline JRCSF.JB WT & $<50$ & $<50$ & $<50$ & $<50$ & $<50$ & $<50$ & $<50$ & $<50$ & $<50$ \\
\hline JRCSF_N156A & $<50$ & $<50$ & $<50$ & $<50$ & $<50$ & $<50$ & $<50$ & $<50$ & $<50$ \\
\hline JRCSF_N133A_N137A & $<50$ & $<50$ & $<50$ & $<50$ & $<50$ & $<50$ & $<50$ & $<50$ & $<50$ \\
\hline JRCSF_N133A_N137A_N156A & $<50$ & $<50$ & $<50$ & $<50$ & $<50$ & $<50$ & $<50$ & $<50$ & $<50$ \\
\hline JRCSF_N332A & $<50$ & $<50$ & $<50$ & $<50$ & $<50$ & $<50$ & $<50$ & $<50$ & $<50$ \\
\hline BG505/T332N & $<50$ & $<50$ & $<50$ & $<50$ & $<50$ & $<50$ & $<50$ & $<50$ & $<50$ \\
\hline BG505/T332N_N156A & $<50$ & $<50$ & $<50$ & $<50$ & $<50$ & $<50$ & $<50$ & $<50$ & $<50$ \\
\hline BG505/T332N_N133A_N137A & $<50$ & $<50$ & $<50$ & $<50$ & $<50$ & $<50$ & $<50$ & $<50$ & 99 \\
\hline MuLV & $<50$ & $<50$ & 78 & $<50$ & $<50$ & $<50$ & $<50$ & $<50$ & $<50$ \\
\hline
\end{tabular}

c

\begin{tabular}{|c|c|c|c|c|c|c|c|}
\hline \multirow{3}{*}{ Virus ID } & \multirow{2}{*}{\multicolumn{7}{|c|}{ ID50 in TZM-bl cells }} \\
\hline & & & & & & & \\
\hline & $\mathrm{Ab893}_{\mathrm{NHP}}$ & $\mathrm{Ab876}_{\mathrm{NHP}}$ & Ab935 $_{\mathrm{NHP}}$ & Ab934 $4_{\mathrm{NHP}}$ & Ab936 $6_{\mathrm{NHP}}$ & Ab988 ${ }_{\mathrm{NHP}}$ & Ab1170 $0_{\mathrm{NHP}}$ \\
\hline JRCSF.JB WT & $<20$ & $<20$ & $<20$ & $<20$ & $<20$ & $<20$ & $<20$ \\
\hline JRCSF_N156A & $<20$ & $<20$ & $<20$ & $<20$ & $<20$ & $<20$ & $<20$ \\
\hline JRCSF_N133A_N137A & $<20$ & $<20$ & $<20$ & $<20$ & $<20$ & $<20$ & $<20$ \\
\hline JRCSF_N133A_N137A_N156A & $<20$ & $<20$ & $<20$ & $<20$ & $<20$ & $<20$ & $<20$ \\
\hline JRCSF_N332A & $<20$ & $<20$ & $<20$ & $<20$ & $<20$ & $<20$ & $<20$ \\
\hline BG505/T332N & $<20$ & $<20$ & $<20$ & $<20$ & $<20$ & $<20$ & $<20$ \\
\hline BG505/T332N_N156A & $<20$ & $<20$ & $<20$ & $<20$ & $<20$ & $<20$ & $<20$ \\
\hline BG505/T332N_N133A_N137A & $<20$ & $<20$ & $<20$ & $<20$ & $<20$ & $<20$ & $<20$ \\
\hline MuLV & $<20$ & $<20$ & $<20$ & $<20$ & $<20$ & $<20$ & $<20$ \\
\hline
\end{tabular}

a, Results of neutralization assays in TZM-bl cells using the serum of eight macaques primed with VLP-RC1-4fill and the serum from a naive macaque at 1:25 dilution against two tier- 2 HIV-1 isolates. ID50, half-maximum inhibitory dose; NHP, non-human primate. b, Results of neutralization assays in TZM-bl cells using the serum from eight macaques primed with VLP-RC1-4fill at 1:50 dilution against the fully glycosylated and glycan-deleted JRCSF.JB and BG505/T332N HIV-1 isolates. c, Results of neutralization assays in TZM-bl cells using monoclonal antibodies isolated from macaques

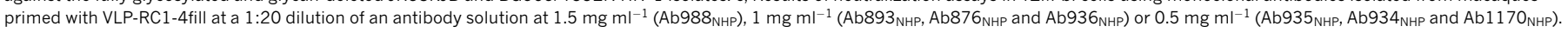
Values are the serum dilution at which relative luminescence units were reduced to $50 \%$ compared to virus control wells (no test sample). 
Extended Data Table 4 | Mouse monoclonal antibodies

\begin{tabular}{|c|c|c|c|c|c|c|c|c|c|}
\hline ANTIBODY & MOUSE & IMM. & VH & CDRH3 & LENGTH (AA) & VK & CDRL3 & LENGTH (AA) & RC1 BINDING \\
\hline 271 & 1 & RC1 & IGHV5-6*01 & ARHSRTGTGAMDY & 13 & IGKV3-4*01 & QQSNEDPPWT & 10 & YES \\
\hline 340 & 2 & $\mathrm{RC} 1$ & IGHV1-81*01 & ARPYYYGSSPYFDY & 14 & IGKV4-57*01 & QQRSSYPPT & 9 & NO \\
\hline 341 & 2 & RC1 & IGHV5-17*01 & ARSIVPDY & 8 & IGKV14-100*01 & VQYVQFPLT & 9 & YES* \\
\hline 343 & 2 & RC1 & IGHV5-6*01 & ASLYGNAFDY & 10 & IGKV3-4*01 & QQSNEDPFT & 9 & YES \\
\hline 344 & 2 & RC1 & IGHV9-3*01 & ASGGNYFDY & 9 & IGKV14-111*01 & LQYDEFPPFT & 10 & YES \\
\hline 346 & 2 & RC1 & IGHV5-6*01 & ARHVGDHAMDY & 11 & IGKV3-4*01 & QQSNEDPFT & 9 & YES \\
\hline 347 & 2 & RC1 & IGHV1-81*01 & ARPYYYGSSPNFDY & 14 & IGKV3-4*01 & QQSNEDPWT & 9 & NO \\
\hline 351 & 3 & RC1 & IGHV9-3*01 & GTGKNYFDH & 9 & IGKV14-111*01 & LQYDEFPYT & 9 & YES \\
\hline 352 & 3 & RC1 & IGHV5-6*01 & ATNYGAWFPY & 10 & IGKV3-4*01 & QQSNEDPYT & 9 & YES \\
\hline 274 & 4 & RC1 & IGHV5-6*01 & ARHGITTVGVAMDY & 14 & IGKV3-4*01 & QQSNEDPWT & 9 & YES \\
\hline 275 & 4 & $\mathrm{RC} 1$ & IGHV5-6*01 & ARHGITTVGVAMDY & 14 & IGKV3-4*01 & QQSNEDPYT & 9 & YES \\
\hline 276 & 6 & RC1-4 & IGHV5-6*01 & ARHGRLTGTGAMDY & 14 & IGKV3-4*01 & QQSNEDPPWT & 10 & YES \\
\hline 278 & 6 & RC1-4 & IGHV5-6*01 & ARHGRLTGTGAMDY & 14 & IGKV3-4*01 & HQSNEDPPWT & 10 & YES \\
\hline 280 & 6 & RC1-4 & IGHV5-6*01 & ARHGHYYGSSYGMDY & 15 & IGKV3-4*01 & QQSNEDPPWT & 10 & YES \\
\hline 294 & 6 & RC1-4 & IGHV2-9*01 & ANIPKDRLCYG & 11 & IGKV3-4*01 & QQSNEDPWT & 9 & YES \\
\hline 348 & NS & RC1 & IGHV1-62-2*01 & ARHEGNYLYAMDY & 13 & IGKV4-62*01 & QQCSGYPLT & 9 & YES \\
\hline 349 & NS & $\mathrm{RC} 1$ & IGHV1-7*01 & ARPPFITVVANYFDY & 15 & IGKV10-94*01 & QQYSKLPWT & 9 & YES \\
\hline
\end{tabular}

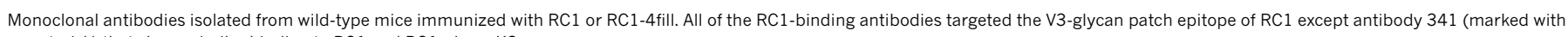
an asterisk) that shows similar binding to RC1 and RC1-glycanKO. 


\section{RESEARCH ARTICLE}

Extended Data Table 5 | Immunoglobulin genes and CDRL3 amino acid sequences of V3-glycan patch bNAbs

\begin{tabular}{lccll}
\hline \multicolumn{1}{c}{ bNAb } & VH & VL & CDRL3 (MT) & CDRL3 (iGL) \\
\hline PGT121 & $4-59$ & L3-21 & HIWDSRVPTKWV & QVWDSSSDHPWV \\
PGT122 & $4-59$ & L3-21 & HIWDSRRPTNWV & QVWDSSSDHPWV \\
PGT123 & $4-59$ & L3-21 & HIYDARGGTNWV & QVWDSSSDHPWV \\
10-1074 & $4-59$ & L3-21 & HMWDSRSGFSWS & QVWDSSSDHPWV \\
PGT124 & $4-59$ & L3-21 & MWDSRSGFSWS & QVWDSSSDHPWV \\
BG18 & $4-4$ & L3-25 & QSSDTSDSYKM & \\
PGT125 & $4-39$ & L2-8 & GSLVGNWDVI & SSYAGSNXXX \\
PGT126 & $4-39$ & L2-8 & SSLVGNWDVI & SSYAGSNXXX \\
PGT127 & $4-39$ & L2-8 & SSLVGNWDVI & SSYAGSNXXX \\
PGT128 & $4-39$ & L2-8 & GSLVGNWDVI & SSYAGSNXXX \\
PGT130 & $4-39$ & L2-8 & SSLFGRWDVV & SSYAGSNXXX \\
PGT131 & $4-39$ & L2-8 & SSLSGRWDIV & SSYAGSNXXX \\
DH270.6 & $1-2$ & L2-23 & SFGGSATVV & SYAGSSTVI \\
\hline
\end{tabular}

Amino acid sequences of the mature (MT) and inferred germline (iGL) CDRL3 of several V3-glycan patch human bNAbs. 


\section{nature research}

Michel C. Nussenzweig and Pamela J.

Corresponding author(s): Bjorkman

Last updated by author(s): Apr 17, 2019

\section{Reporting Summary}

Nature Research wishes to improve the reproducibility of the work that we publish. This form provides structure for consistency and transparency in reporting. For further information on Nature Research policies, see Authors \& Referees and the Editorial Policy Checklist.

\section{Statistics}

For all statistical analyses, confirm that the following items are present in the figure legend, table legend, main text, or Methods section.

n/a Confirmed

$\square$ \The exact sample size $(n)$ for each experimental group/condition, given as a discrete number and unit of measurement

$\square$ \A statement on whether measurements were taken from distinct samples or whether the same sample was measured repeatedly

$\triangle$ The statistical test(s) used AND whether they are one- or two-sided

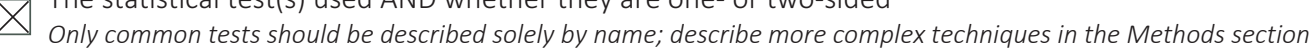

$\square$ \A description of all covariates tested

$\square$ \A description of any assumptions or corrections, such as tests of normality and adjustment for multiple comparisons

$\square$ A full description of the statistical parameters including central tendency (e.g. means) or other basic estimates (e.g. regression coefficient)

$\triangle$ AND variation (e.g. standard deviation) or associated estimates of uncertainty (e.g. confidence intervals)

$\square$ For null hypothesis testing, the test statistic (e.g. $F, t, r$ ) with confidence intervals, effect sizes, degrees of freedom and $P$ value noted

Give P values as exact values whenever suitable.

Х $\square$ For Bayesian analysis, information on the choice of priors and Markov chain Monte Carlo settings

Х $\square$ For hierarchical and complex designs, identification of the appropriate level for tests and full reporting of outcomes

$\square$ \Estimates of effect sizes (e.g. Cohen's d, Pearson's $r$ ), indicating how they were calculated

Our web collection on statistics for biologists contains articles on many of the points above.

\section{Software and code}

Policy information about availability of computer code

Data collection ELISA data was collected using the software Omega.LNK. Flow cytometry data was collected using FACSDiva version 8.0.2 EPU automated data acquisition software (Thermo Fisher). Bio-layer Inferometry data was collected by Data aquisition 10.0 (FORTEBIO)

Data analysis

MacVector 15.5.3 was used for sequence analysis and graphs were created using R language. Flow cytometry data was processed using FlowJo 10.5.0. GraphPad Prism 7 was used for data analysis. Ig gene sequence AB1 files were converted to FASTQ format using biopython package. FASTQ files were trimmed by quality using cutadapt v1.18 software. Igblast v1.9.0 was used for VDJ assignment and clone analysis was performed using Change-O software v0.3.7. For macaques, a custom VDJ database was created using previously reported Ig gene sequences. Bio-layer inferometry data was analysed using Data analysis HT 10.0 (FOREBIO). SPR from Biacore T200 software v3.0. Phenix v1.14, Coot v0.8.9, RELION v3.0, cryoSPARC v2.2, PyMOL v2.1, Chimera v1.13, , Molprobity v4.4, Resmap v1.1.4, and Privateer v1 were used for structural analysis.

For manuscripts utilizing custom algorithms or software that are central to the research but not yet described in published literature, software must be made available to editors/reviewers. We strongly encourage code deposition in a community repository (e.g. GitHub). See the Nature Research guidelines for submitting code \& software for further information.

\section{Data}

Policy information about availability of data

All manuscripts must include a data availability statement. This statement should provide the following information, where applicable:

- Accession codes, unique identifiers, or web links for publicly available datasets

- A list of figures that have associated raw data

- A description of any restrictions on data availability

Cryo-EM reconstructions of the RC1-10-1074, RC1-Ab275mur, RC1-Ab874nhp, and RC1-897nhp complexes have been deposited in the Electron Microscopy Data Bank under the accession numbers AAAA, BBBB, CCCC, and DDDD, respectively. Coordinates for atomic models of the RC1-10-1074, RC1-Ab275mur, RC1-Ab874nhp, and RC1-897nhp complexes have been deposited in the Protein Data Bank under the accession numbers WWWW, XXXX, YYYY, and ZZZZ, respectively. (Accession 


\section{Field-specific reporting}

Please select the one below that is the best fit for your research. If you are not sure, read the appropriate sections before making your selection. $\bigotimes$ Life sciences $\quad \square$ Behavioural \& social sciences $\quad \square$ Ecological, evolutionary \& environmental sciences

For a reference copy of the document with all sections, see nature.com/documents/nr-reporting-summary-flat.pdf

\section{Life sciences study design}

All studies must disclose on these points even when the disclosure is negative.

Sample size Groups of 3-5 mice were used for immunizations considering assay variability . 4 rabbits and 8 Rhesus macaques. No statistical analysis was performed to predetermine sample size but these are standard numbers for the field.

Data exclusions No data were excluded

Replication Immunization experiments in wild type mice were performed more than 3 times. Antibody binding to Env was confirmed using different methods: ELISA, OCTET, SPR and/or Cryo-EM. All attempts at replication were successful.

Randomization $\quad$ C57BL/6J wild type mice were purchased from The Jackson laboratory and divided in groups of same age and sex.

Blinding Mice were homogenous in sex and age prior to grouping. Blinding is not relevant in this study. Animal samples were analysed using objective, standardized assays that include appropriate controls. There is no subjective assessment of the animals.

\section{Reporting for specific materials, systems and methods}

We require information from authors about some types of materials, experimental systems and methods used in many studies. Here, indicate whether each material, system or method listed is relevant to your study. If you are not sure if a list item applies to your research, read the appropriate section before selecting a response.

Materials \& experimental systems

n/a Involved in the study

$\square$ \ Antibodies

$\square \bigotimes$ Eukaryotic cell lines

$\bigotimes \square$ Palaeontology

$\square \bigotimes$ Animals and other organisms

$\bigotimes \square$ Human research participants

$\bigotimes \square$ Clinical data

\begin{tabular}{l|l}
\multicolumn{2}{l}{ Methods } \\
\hline n/a & Involved in the study \\
$\square$ & $\square$ ChIP-seq \\
$\square$ & $\bigotimes$ Flow cytometry \\
$\square$ & $\square$ MRI-based neuroimaging
\end{tabular}

\section{Antibodies}

Antibodies used

Anti-mouse IgG (Jackson ImmunoResearch \#115-035-071), anti-human IgG heavy chain (Jackson ImmunoResearch \#109-035-098) or anti-human Ig heavy and light chain (Jackson ImmunoResearch \#109-036-088) conjugated to horseradish peroxidase (HRP) were used in ELISAS.

Mouse (BD Biosciences \#553142) and human (BD Biosciences \#564219) Fc Block. anti-CD4 APC-eFluor780 (Invitrogen, \#47-0042-82, cloneRM4-5, Lot \# 1994174), anti-CD8 APC-eFluor780 (Invitrogen, \#47-0081-82, clone 53-6.7, Lot\# 1989142), anti-F4/80 APC-eFluor780 (Invitrogen, \#47-4801-82, clone BM8, Lot\# 4338513), antiNK1.1 APC-eFluor780 (Invitrogen, \#47-5941-82, clone PK136, Lot\#1983611), anti-CD11b APC-eFluor780 (eBioscience \#47-0112-82, clone M1/70, Lot\#4341634), anti-CD11c APC-eFluor780 (eBioscience \#47-0114-82, clone N418, Lot\#E10192-1633), anti-Gr-1 APC-eFluor780 (Invitrogen, \#47-5931-82, clone RB6-BC5, Lot\#1994248), anti-B220 APC (eBioscience, \#17-0452-82, clone RA3-6B2, Lot\#E07151-1635), anti-GL7 FITC (BD Biosciences \#553666, clone GL7, Lot\#6021860) and anti-CD95 BV421 (BD Biosciences \#562633, clone Jo2, Lot\#4234912) were used for cytometric analysis of mouse B cells. anti-CD16 APC-eFluor780 (Invitrogen, \#47-0168-41, clone eBioCB16 (CB16), Lot\#1942879), anti-CD8a APC-eFluor780 (Invitrogen, \#47-0086-42, clone OKT8 (OKT-8), Lot\#1946503), anti-CD3 APC-eFluor780 (Invitrogen, \#47-0037-41, clone OKT3, Lot\#1915545), anti-CD14 APC-eFluor780 (eBiosciences, \#47-0149-41, clone 61D3, Lot\#4319282), anti-CD20 PeCy7 (BD, \#335793, clone , Lot\#8204544), anti-CD38 FITC (Stem Cell technologies, \#60131FI, clone AT-1, Lot\#18A86904), anti-IgG BV421 (BD Biosciences, \#562581, clone G18-145, Lot\#7355875), anti-IgM PerCP-Cy5.5 (BD Biosciences, \#561285, clone G20,-127, Lot\#7278582) were used for cytometric analysis of macaque B cells.

All antibodies were used at 1:200 dilution. 


\section{Eukaryotic cell lines}

Policy information about cell lines

Cell line source(s)

HEK293-6E cells (National Research Council of Canada)

Expi293F cells (Thermo Fisher)

E. coli C41(DE3) cells (Novagen)

Authentication

Cell lines were obtained from and authenticated by vendors or scientific collaborators.

Mycoplasma contamination

Commonly misidentified lines

(See ICLAC register)

The cell lines were not contaminated by mycoplasma as determined by using the Lonza Mycoplasma Detection Kit.

None

\section{Animals and other organisms}

Policy information about studies involving animals; ARRIVE guidelines recommended for reporting animal research

Laboratory animals

Mice carrying the Ig V(D)J genes encoding the $\mathrm{IGL}$ IgH and IgL corresponding to the human PGT121 and 10-1074 broadly neutralizing antibodies (GLHL121 knock-in mice) were used for immunizations. C57BL/6J male mice from The Jackson laboratory were used for immunizations. Male and female mice of 6-8 weeks of age were used for experiments.

Six-month-old New Zealand White rabbits (Covance) were used for immunizations.

Sixteen male and female rhesus macaques (Macaca mulatta) of Indian genetic origin of 2 to 4 years of age were used for immunizations.

Wild animals

This study does not involve wild animals.

Field-collected samples

For laboratory work with field-collected samples, describe all relevant parameters such as housing, maintenance, temperature, photoperiod and end-of-experiment protocol OR state that the study did not involve samples collected from the field.

Ethics oversight

All procedures in mice were performed in accordance to protocols approved by the Rockefeller University IACUC. All procedures performed in rabbits were done by Covance and approved by Denver PA IACUC Committee, \#0003-18. Rhesus macaques were housed and cared for in accordance with Guide for Care and Use of Laboratory Animals Report no. NIH 82-53 (Department of Health and Human Services, Bethesda, Maryland, 1985) in a biosafety level $2 \mathrm{NIH}$ facility. All animal procedures and experiments were performed according to protocols approved by the Institutional Animal Care and Use Committee of NIAID, NIH.

Note that full information on the approval of the study protocol must also be provided in the manuscript.

\section{Flow Cytometry}

\section{Plots}

Confirm that:

\The axis labels state the marker and fluorochrome used (e.g. CD4-FITC).

$\bigotimes$ The axis scales are clearly visible. Include numbers along axes only for bottom left plot of group (a 'group' is an analysis of identical markers).

$\bigotimes$ All plots are contour plots with outliers or pseudocolor plots.

$\bigotimes$ A numerical value for number of cells or percentage (with statistics) is provided.

\section{Methodology}

Sample preparation

Instrument

Software
Single cell suspensions were obtained from the draining lymph nodes and spleens of immunized mice, and mature B-cells were isolated by negative selection using anti-CD43 magnetic beads (MACS) following the manufacturer's instructions.

Frozen PBMCs or cells from lymph node biopsies obtained from the naïve and immunized macaques were thawed and washed in RPMI medium 1640 (1x) (Gibco \#11875-093).

BD FACSAria II

FACSDiva version 8.0.2. 
Gating strategy

Single RC1 positive RC1-glycanKO negative mouse B cells were isolated by gating on single cells, Live/dead marker (Zombi NIR) negative, CD4 negative, CD8 negative, F4/80 negative, NK1.1 negative, CD11b negative, CD11c negative, Gr-1 negative, B220 positive, CD95 positive, GL7 positive, RC1 positive and RC1-glycan KO negative.

Single macaque cells binding to RC1 but not to RC1-glycanKO were isolated by gating on single cells, Live/dead marker (Zombi NIR) negative, CD16 negative, CD8a negative, CD3 negative, CD14 negative, CD20 positive, CD38 positive, IgG positive and negative, IgM positive and negative, RC1 positive and RC1-glycanKO negative.

\Tick this box to confirm that a figure exemplifying the gating strategy is provided in the Supplementary Information. 